\title{
IMPLANTATION OF CARBON IN GaAs
}

\author{
AMY JO MOLJ, \\ M.S. Thesis \\ MATERIALS SCIENCE AND MINERAL ENGINEERING DEPARTMENT \\ University of California \\ and \\ MATERIALS SCIENCES DIVISION \\ CENTER FOR ADVANCED MATERIALS \\ Lawrence Berkeley Laboratory \\ University of California \\ Berkeley, CA 94720
}

MARCH 1992

This work was supported by the Director, Office of Energy Research, Office of Basic Energy Sciences, Matcrials Sciences Division, of the U.S. Department of Energy under Contract No. DE-AC03-76SF00098. 


\section{Implantation of Carbon in GaAs}

by

Amy J. Moll

\section{ABSTRACT}

Carbon implanted into GaAs and thermally annealed typically exhibits very low $(<3 \%)$ electrical activity. It has been demonstrated that the electrical activity of $C$ can be significantly enhanced by co-implantation with Ga. Improved activation may result from either additional damage of the crystal lattice or from stoichiometric changes, forcirg the $\mathrm{C}$ atoms onto As sites. To determine the relative importance of each of these effects, I have undertaken a systematic study of carbon activation in GaAs. A range of co-implants have been used: group III (B, Ga), group $V(N, P, A s)$ and noble gases $(A r, K r)$. The damage introduced to the substrate will depend on the mass of the ion implanted. The group $I I I$ and group $V$ co-implants will affect the crystal stoichiometry. The results indicate that both lattice damage and crystal stoichiometry are important for high electrical activity of C. Increasing the damage will increase the activation due to the increased number of As vacancies but maximum activation can be obtained only by a co-implant which not only damages the lattice but also forces the $C$ to occupy an As site. 


\section{ACKNOWLEDGMENTS}

I would like to thank the many people who have contributed to the successful completion of this thesis. Eugene Haller has given me the opportunity to conduct this research and has provided continuing support and guidance. Bill Hansen, Kin Man Yu, John Madok, and Kevin Roderick have made significant contributions to my research. Special thanks to Wladek Walukiewicz for many hours of discussion and his helpful comments on my thesis and to Prof. Nathan Cheung for reviewing my thesis.

I would like to thank the Office of Naval Research for their financial support.

In addition I would like to thank all members of the electronic materials group. Each one has always been willing to share his or her knowledge and skills. Most of all I enjoy the camaraderie in the group which makes coming to work fun.

Last, but certainly not least, thank you to my parents without whom I would not be where I am today. 
Acknowledgments

1. Introduction

2. Ion Implantation Fundamentals

6

2.1 Range and Stopping of Implanted lons

2.2 Radiation Damage

2.3 Channeling

3. Ion Implantation in GaAs

3.1 Typical Dopants

3.2 Maintaining Stoichiometry

3.3 Implantation Damage in GaAs

4. Annealing

4.1 General Requirements

4.2 Damage Removal

4.3 Protection of Surface

4.4 Rapid Thermal Annealing

5. Carbon in GaAs

5.1 Properties of $C$ in GaAs

5.2 Ion Implantation of $C$ in GaAs

5.3 Effect of Ga Co-implant

6. Experimental

7. Results

7.1 Electrical Characterization

7.2 Structural Characterization

8. Discussion

9. Conclusions

10. References

Appendices

I. Summary of results of carbon implantation reported in the literature 71

II. Calculated concentration profiles for implanted ions

III. Complete electrical data from C implantation 


\section{INTRODUCTION}

The stopping of energetic ions in matter has been a subject of theoretical and experimental interest since the early 1900's and the discovery of energetic particle emission from radioactive elements. The fundamental work in this field was done by Rutherford 1 and Bohr ${ }^{2}$ and includes theories on the range distribution of implanted ions, energy loss of lons during implantation, distribution of the radiation damage produced by irradiation, as well as theories on the different ion penetration behavior in amorphous versus crystalline solids. The use of ion implantation in the semiconductor industry was developed in 1950 's and 1960's when its potential for introducing dopants in semiconductors in a controlled fashion was recognized. By the early 1970's most major organizations developing and manufacturing electronic devices were using or exploring ion implantation. ${ }^{3}$

During the process of ion implantation, atoms or molecules are ionized, mass separated, and accelerated in an electrostatic field, and implanted into a solid. Almost any kind of ion can be irnplanted into any solid. In the traditional doping process used by the serniconductor industry, boron or phosphorus ions are implanted in silicon. However, in many other materials systems such as ceramics and metals, ion implantation is increasingly used for materials modification. The acceleration energy of the ions varies from hundreds of electron volts to several millions electron volts. The penetration depth of the ions depends on the energy, the mass of the ion and the atomic mass of the substrate. Depths of tens of nanometers to several microns are typical for these energies. In general, the atoms introduced have a concentration profile which can be described by a Gaussian distribution, with an average projected range $R_{p}$ and a standard deviation $\Delta R_{p}$ parallel to the implantation direction and $\Delta R_{p L}$ corresponding to the average lateral straggle of the implanted ions. 
implantation direction and $\Delta \mathrm{R}_{\mathrm{pL}}$ corresponding to the average lateral straggle of the implanted ions.

In the semiconductor industry, ion implantation is used as a standard doping technology. Other doping technologies include: doping during crystal growth, diffusion from a surface or near surface source, and alloying. Ion implantation offers several technological advantage ${ }^{4}$ over other forms of doping. Principally, these are the throughput, homogeneity and reproducibility of the doping process, and the exact control of the number of dopant atoms introduced. In comparison with other doping techniques, ion implantation has lower requirements for the purity of the dopant source. Within the implanter the components of the source beam are separated according to their mass to charge ratio.

During implantation the temperature of the substrate can be controlled. A wide range of temperatures have been used. Low substrate temperatures during implantation inhibits the diffusion of impurities and defects present in the substrate. Simple masking methods aliow for the doping of specific areas of the substrate. Layers of oxide, nitride, metal or photoresist several microns thick are commonly used as masks during implantation. Implantation can be performed through thin layers (e.g., $\mathrm{SiO}_{2}, \mathrm{Si}_{3} \mathrm{~N}_{4}$ ). The small penetration depth of the ions (in general, less than a few microns) results in shallow layers with very high dopant concentration gradients. Shallow doped layers allow for the fabrication of devices with very small dimensions which is increasingly important for VLSI and ULSI production. Through multiple implantations at varying energies, the doping profile can be tailored to particular device requirements.

The extent of the application of ion implantation in the semiconductor industry is limited by certain disadvantages associated with the technique. For 
a particular application the following issues must be considered to determine the applicability of ion implantation. 4 Most of the implanted atoms do not come to rest on substitutional sites and therefore are electrically inactive. In addition, bombardment with heavy particles produces lattice damage. In general, the radiation damage results in deterioration of the semiconductor's electrical properties. Thermal annealing is required to create substitutional, electrically active impurities and to restore crystallinity. The high temperatures (>600 C) which are required may lead to diffusion of dopants present in the substrate compensating the implanted dopants.

The depth of implanted dopants is limited to near surface layers (less than a few microns). Greater penetration depths can be achieved by implanting at higher accelerating voltages however it is difficult to recover a perfect crystal particularly near the projected range, $R_{p}$. The residual damage results in unsatisfactory electrical properties of the implanted layers. Another method of doping thick layers is to implant dopants then allow them to diffuse deeper into the substrate by processing at high temperatures. Another difficulty in devices created by ion implantation is the inaccuracy of theoretically predicted implant profiles. Random channelling of the dopant ions results in deeper penetration while diffusion during annealing can result in both deeper penetration as well as lateral spreading.

The typical ion implantation machine can implant elements with atomic weights ranging from 1 amu to 130 amu. Energies can be varied between 25 and $200 \mathrm{keV}$ for singly ionized atoms and between 200 and $400 \mathrm{k} \theta \mathrm{V}$ for doubly ionized atoms. The corresponding range of the ions for the above parameters lie between 50 and $500 \mathrm{~nm}$ (depending on the mass of the implanted ions and the density of the substrates). Typical doses for semiconductor device applications range from $1 \times 10^{12} \mathrm{~cm}^{-2}$ to $1 \times 10^{16} \mathrm{~cm}^{-2}$. 
1) Room temperature implantation.

2) Low mass ions. Lower mass allows for a greater depth range of the ions while kecping within reasonable values of implantation energies. Less damage occurs when implanted ions are of lower mass and thus a annealing can occur at a lower temperature.

3) Low temperature anneal. Higher temperatures will encourage diffusion of impurities, contamination, and promote degradation of the surface through loss of As.

4) Easily attainable source compounds.

5) Non-toxic sources.

6) High activation efficiency. Activation efficiency is the percentage of implanted ions which become electrically active dopants. It can be determined by the ratio of sheet free carrier concentration to implant dose.

7) High iree carrier mobility in the implanted layers.

This thesis discusses the implantation of carbon in GaAs. As an introduction, the fundamentals of ion implantation will be described in chapter 2. Chapter 3 discusses ion implantation in GaAs specifically the additional complications which resulf from implantation into a compound semiconductor rather than an elemental semiconductor such as $\mathrm{Si}$. The ideal conditions described are not easily met for implantation in GaAs as explained in this chapter. Annealing of radiation damage and activation of implanted ions will be discussed in Chapter 4.

Chapter 5 is a review of the literature regarding carbon implantation in GaAs. C implantation is of particular interest since after annealing only a small percentage of implanted ions contribute a free carrier. Co-implantation of $\mathrm{Ga}$ results in a higher percentage of $C$ ions becoming electrically active. However, the exact role of the $\mathrm{Ga}$ co-implant is unclear, it may act to maintain 
stoichiometry in the implanted layer or to create additional radiation damage in the substrate. The experimental work presented in this thesis examines this issue by separating the effects of damage and stoichiometry by co-implanting group III, group V and group VIII ions. The experimental approach is described in chapter 6 ; results are presented in chapter 7; and chapter 8 is a discussion of the results. 


\section{ION IMPLANTATION FUNDAMENTALS}

\subsection{Range and Stopping of Implanted lons}

lons implanted into solid substrates are slowed and eventually stopped principally by two different mechanisms:

1. Inelastic collisions, also called "electronic stopping." The energetic ion gives energy to the electrons of the substrate, resulting in ionization or excitation of the host atom.

2. Elastic collisions, also called "nuclear stopping." The incoming ion collides with nuclei of the substrate. A fraction of the kinetic energy of the incoming ion is transferred to the nuclei of the substrate.

In this section, a brief description of the basic physical concepts involved in these stopping mechanisms will be presented. The theoretical principles used in the calculation of the range of implanted ions are found in nuclear physics. These foundations were investigated by Bohr and Rutherford in their work on the interaction of energetic particles with solid targets.

Nuclear stopping can be considered as a collision between two point masses $\left(M_{1}\right.$ for the incident or moving ion and $M_{2}$ for the struck particle or nucleus). The classical mechanical dynamic equations of motion under a central force constraint can then be set up and solved. Total energy and total momentum are conserved. The end result of this elastic collision requires that all energy lost by the incident particle is acquired by the struck particle. In this description, the detailed electronic structure of the interacting particles is averaged to construct a total force and potential function such that each particle is treated as a point mass.

The force acting between the two particles is Coulombic: 


$$
F(r)=\frac{Z_{1 \Theta H} Z_{2 e f f} \theta^{2}}{4 \pi \varepsilon_{0} r^{2}}
$$

where $Z_{1 \text { eff }}$ and $Z_{\text {2eff }}$ take into account the screening of the nuclear charge by the electrons. The potential of interaction is the spatial integral of the force:

$$
V(r)=\frac{Z_{1 \text { eff }} Z_{2 \text { eff }} e^{2}}{4 \pi \varepsilon_{0} r}
$$

The force and the potential decrease rapidly with increasing atomic separation, varying as $1 / r^{2}$ and $1 / r$ respectively.

In ion implantation the most distant collisions between an ion and a target atom will occur for an ion incident at a distance no greater than about half the interatomic lattice spacing in a crystalline target. The closest collisions will occur for an ion directly incident upon a target atom, a head-on collision The struck atom moves away from the incident ion during the whole period, and the minimum distance of closest approach is about $.01 \mathrm{~nm}$. Because the range of interaction distances is very small and within this range the potential falls steeply with increasing separation, a first order approximation of the potential is to assume that $V(r)$ is a step function, i.e., $V(r)$ is a constant from $r=0$ to some distance $r=r_{0}$ at which $V(r)$ falls to 0 . This approximation results in each atom being treated as a perfectly elastic hard sphere of radius $r_{0}$ where no interaction occurs for $>r_{0}$. The hard sphere model is reasonably accurate for near headon atomic collisions where the range of separation values over which strong interaction occurs, is limited.

Consider as in Figure 2.1, the collision of two hard spheres, each of radius $r_{0}$ with the incident sphere of mass $M_{1}$ and energy $E_{0}$ and the struck atom of mass $M_{2}$, initially at rest. The closest distance of approach for the centers of the two atoms is $2 r_{0}$ but the perpendicular distance between the initial direction of motion of the incident ion and the parallel line through the 


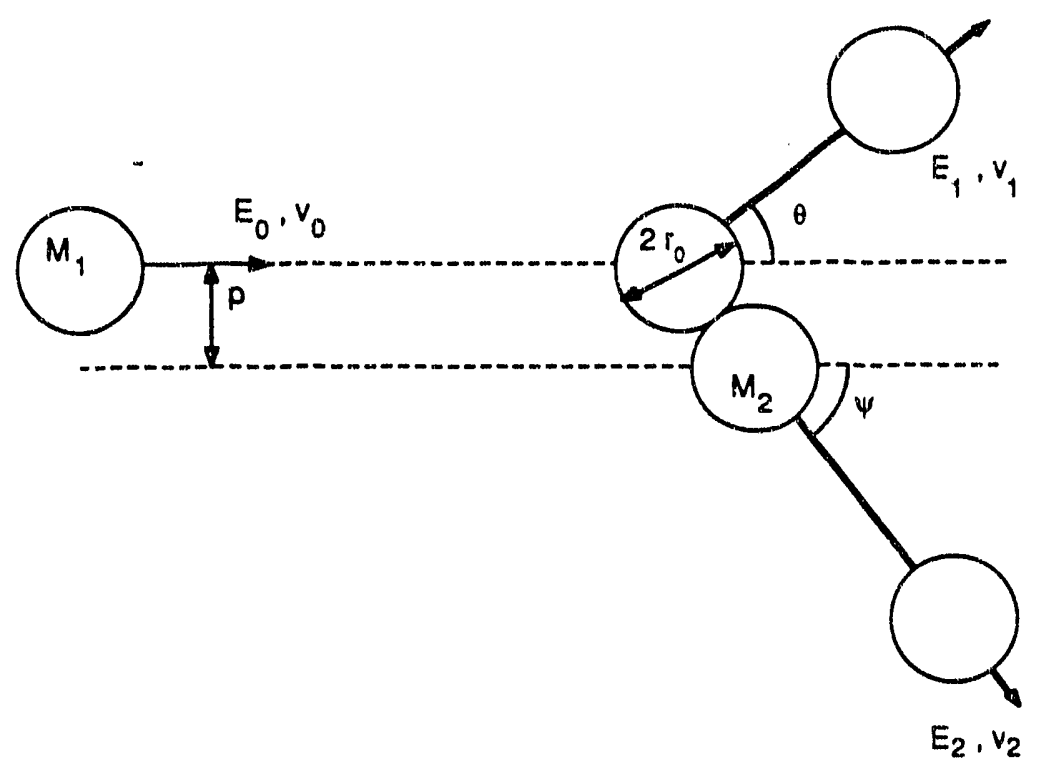

Figure 2.1. The geometry of a collision between two hard elastic spheres.

initial position of the center of the struck atom will be less than $2 r_{0}$. This perpendicular distance, $p$, is known as the impact parameter for the collision.

In the collision, kinetic energy is conserved and momentum is transferred along the line of the centers of the particles. The struck atorn moves at an angle $\psi$ to the initial direction of the particle motion and parallel to the line of the centers. Before the collision the incident particle velocity is $v_{0}$ and after the collision its velocity is $v_{1}$. It is moving at an angle $\theta$ to its initial direction of motion while the struck atom moves away from the collision with a velocity $v_{2}$. The equations for the conservation of energy and momentum are then given by. energy: $\quad \frac{1}{2} M_{1} v_{0}^{2}=E_{0}=\frac{1}{2} M_{1} v_{1}^{2}+\frac{1}{2} M_{2} v_{2}^{2}=E_{1}+E_{2}$,

momentum (parallel to incident direction):

$$
M_{1} v_{0}=M_{1} v_{1} \cos \theta+M_{2} v_{2} \cos \psi \text {, }
$$

and momentum (perpendicular to incident direction: 


$$
0=M_{1} v_{1} \sin \theta \cdot M_{2} v_{2} \sin \psi .
$$

These equations can be solved to show that

and

$$
\begin{aligned}
& v_{1}^{2}=v_{0}^{2}\left\{\frac{M_{1} \cos \theta+\left(M_{2}^{2}-M_{1}^{2} \sin ^{2} \theta\right)^{1 / 2}}{M_{1}+M_{2}}\right\} \\
& \cos \theta=\frac{1}{2}\left\{\left(1+\frac{M_{2}}{M_{1}}\right)\left(\frac{E_{2}}{E_{0}}\right)^{\frac{1}{2}}+\left(1-\frac{M_{2}}{M_{1}}\right)\left(\frac{E_{0}}{E_{2}}\right)^{\frac{1}{2}}\right\} .
\end{aligned}
$$

The maximum energy transferred $\left(T_{m}: E_{2}\right)$ occurs when $\theta=\pi$ and is given by

$$
T_{m}=E_{0}\left\{\frac{4 M_{1} M_{2}}{\left(M_{1}+M_{2}\right)^{2}}\right\}
$$

From the geometry of the collision

$$
\sin \psi=\frac{p}{2 r_{0}}
$$

In ion implantation, a flux of ions is incident upon the substrate. Only the ions with impact parameter $p<2 r_{0}$ will collide with a particular atom in the substrate. The area $\pi\left(2 r_{0}\right)^{2}$ defines a total collision cross-section. A flux of ions, each with impact parameter $p$ will be deflected through an angle $\theta$, into a cone of half angle $\theta$ about the center of the struck atom. Similarly, ions incident at impact parameter $p+\delta p$ will be unitormly deflected into a cone of half angle $\theta+$ $\delta \theta$ (see Figure 2.2). The plane area defined by the radii $p$ and $p+\delta p$ defines ions which are scattered between angles $\theta$ and $\theta+\delta \theta$. This area,

$$
\pi\left\{(p+\delta p)^{2}-p^{2}\right\}=d\left(\pi p^{2}\right)=2 \pi p \delta p
$$

is known as the differential scattering cross section, $d \sigma$, for scattering between impact parameters of $p$ and $p+\delta p$ and scattering angles $\theta$ and $\theta+\delta \theta$.

The energy transferred to the substrate atom, $T\left(=E_{2}\right)$ depends on the scattering angle, $\theta$. The differential cross-section do defines the differential cross-section for energy transters in the range $T$ to $T+\delta T$. 


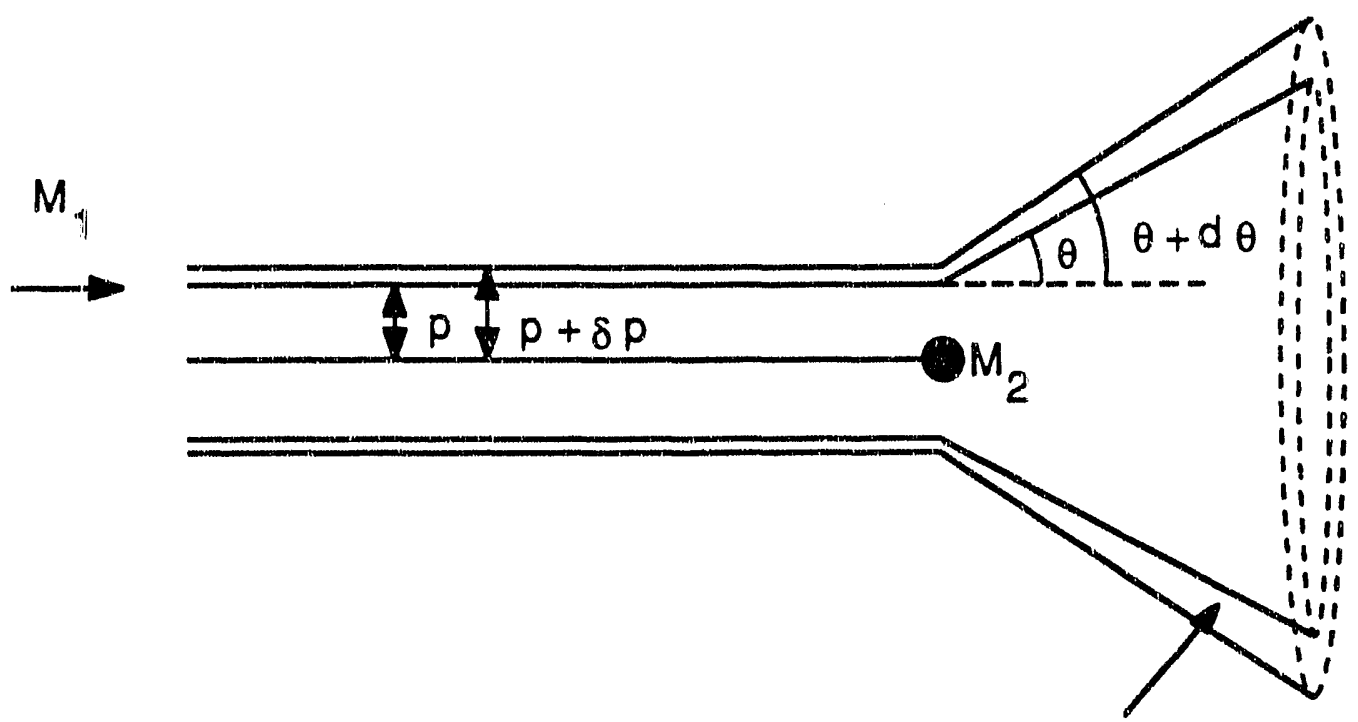

$2 \pi \sin \theta d \theta$

Figure 2.2. Illustration of the differential scattering cross-section.

The energy transfer, $T$ can be defined as a function of the impact parameter $p$

$$
p^{2}=4 r_{0}^{2}\left(1-\frac{T}{T_{m}}\right)
$$

and the differential scattering cross-section

$$
2 \pi p d p=\pi \frac{4 r_{0}^{2}}{T_{m}} d T
$$

Therefore, for hard sphere collisions the differential scattering crosssection for energy transfer between $T+\delta T$ is dependent only on the energy limits $\delta T$ and not on the transferred energy itself. Similarly the probability of a collision with impact parameter between $p$ and $\delta p$ is just

$$
\frac{2 \pi p d p}{4 \pi r_{0}^{2}}=\frac{d T}{T_{m}}
$$


which is independent of energy transfer, impact parameter, and scattering angle. Therefore, in the hard sphere case, all energy transfers and all scattering angles ar equally probable; scattering is isotropic.

In ion implantation, a moving ion experiences a succession of single atomic encounters of the type described above as it interacts with the atoms of the target lattice. At each encounter the ion loses energy and viewed statistically over many collisions ions will on average lose a given amount of energy per unit distance in the solid. This rate of energy loss is written $-d E / d x$ and can be determined formally in terms of the parameters discussed above. If the energy lost by an ion in a single collision is $T$, the average loss of energy in a collision is

$$
\bar{T}=\frac{\int_{0}^{T_{m}} T d \sigma}{\int_{0}^{T_{m}} d \sigma}
$$

If there are $\mathrm{N}$ target atoms per unit volume, randomly spatially distributed relative to the primary ion, then in unit distance travelled by the ions the total number of atomic encounters by the ion is $N \int_{0}^{T_{m}} d \sigma$.

Thus the mean rate of energy loss per unit path length is

$$
\bar{T} N \int_{0}^{T_{m}} d \sigma \quad \text { or } \quad-\frac{d E}{d x}=N \int_{0}^{T_{m}} T d \sigma \text {. }
$$

The above description applies to elastic collisions or "nuclear stopping." The other principle stopping mechanism experienced by implanted ions is due to inelastic collisions with electrons of the substrate. Energy is lost due to electron excitations and ionizations which occur in the substrate atoms as the ion passes. A comprehensive treatment of these inelastic energy exchange processes could only be given by a quantum mechanical approach detailing 
the behavior of each electronic wave function. A qualitative description of these losses will be given here by using a semi-classical approach.

By using the equations above, an estimation of the ion energy required for the excitation of an electron can be calculated. The maximum energy transfer, $T_{m}$, from an ion mass $M_{1}$ and energy $E_{0}$ to an electron of mass $M_{0}$ in a head on collision can be approximated by assuming $M_{1} \gg M_{0}$, setting $M_{2}=M_{0}$, and substituting in equation [2.8].

$$
T_{m}=\frac{4 M_{0}}{M_{1}} E_{0}
$$

If electron excitation is to occur then this energy transfer must exceed some minimum energy for excitation, i.e., $\left(4 M_{0} / M_{1}\right) E_{0}>E_{e}$. Excitation energies are on the order of several $\mathrm{eV}$. Taking $E_{e}=4 \mathrm{eV}$ then $E_{0}>M_{0} / M_{1}(\mathrm{eV})$ furthermore since the mass of the implanted ion, $M_{1}$ is several thousand times the electron mass a rough approximation of the criterion for excitation becomes $E_{0}>M_{1}$ $(\mathrm{keV})$. In this simple approximation, excitation effects assume importance when the ion energy in $\mathrm{keV}$ exceeds $M_{1}$, the numerical value of the atomic mass of the ion. However, this is orily a rough approximation, and inelastic effects are not entirely absent for ion energies below $M_{1} \mathrm{keV}$.

The stopping cross-section $\mathrm{S}$ of the solid is given by

$$
S_{e, n}=-\frac{1}{N}\left(\frac{d E}{d x}\right)_{e, n} e^{2} \mathrm{~cm}^{2}
$$

The subscripts, $e$ and $n$, refer to electronic and nuclear stopping powers respectively. The relative importance of the two stopping mechanisms is determined by the energy and mass of the implanted ions and the mass and atomic derisity of the solid. The stopping power as a function of the square root of the imiplanted ion energy is given in Figure 2.3. Characteristic energies $E_{1}$, $E_{2}$ and $E_{3}$ for some typical implants into GaAs are given in Table 2.1 . 


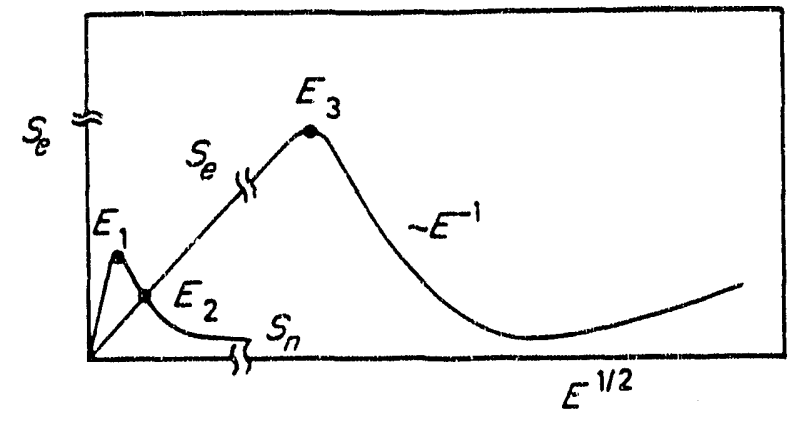

Figure 2.3. Schematic of the relative values of the nuclear $\left(S_{n}\right)$ and electronic $\left(S_{e}\right)$ stopping powers as a function of ion energy. (From Ref. 4.)

Table 2.1. Characteristic energies for some typical implants in GaAs. (From Ref. 4)

\begin{tabular}{c|c|c|c} 
Ion & $E_{1}(\mathrm{keV})$ & $E_{2}(\mathrm{keV})$ & $E_{3}(\mathrm{keV})$ \\
\hline$B$ & 7 & 13 & $3 \times 10^{3}$ \\
$P$ & 29 & 140 & $3 \times 10^{4}$ \\
As & 103 & 800 & $2 \times 10^{5}$
\end{tabular}

Typical implantation energies fall in the range where nuclear stopping is dominant. The rate of energy loss by nuclear collisions per unit distance is given below:

$$
-\frac{\mathrm{dE}}{\mathrm{dx}}=2.8 \times 10^{-15} \mathrm{~N}\left[\frac{\mathrm{Z}_{1} \mathrm{Z}_{2}}{\mathrm{Z}_{1}^{2 / 3}+\mathrm{Z}_{2}^{2 / 3}}\right]\left[\frac{\mathrm{M}_{1}}{\mathrm{M}_{1}+\mathrm{M}_{2}}\right] \frac{\mathrm{eV}}{\mathrm{cm}}
$$


For typical implant conditions $\mathrm{dE} / \mathrm{dx}$ is on the order of $100-1000 \Theta \mathrm{V} / \mathrm{nm}$ for nuclear stopping. The elastic energy given up by the implanted ions is transferred to the recoiling substrate nuclei. If the displaced nucleus has enough energy it can dislodge other atoms leading to a cascade. At high energies $\left(E>E_{2}\right.$ in Figure 2.3) the loss due to nuclear scattering is very small. At intermediate energies the stopping power due to nuclear collisions increases and then at low energies it again falls off due to the screening effects of the electrons resulting in a lower effective atomic number of the target nuclei.

Electronic stopping, $S_{e}$, is proportional to the velocity of the implanted ion and therefore proportional to the square root of the implant energy.

$$
\mathrm{S}_{\mathrm{e}}=\mathrm{k} \sqrt{\mathrm{E}}
$$

For GaAs $\mathrm{k}=0.52 \times 10^{-15}(\Theta \mathrm{V})^{1 / 2} \mathrm{~cm}^{2}$ and therefore $\mathrm{dE} / \mathrm{dx}$ is on the order of 100 's of eV/nm which is approximately the same as for nuclear stopping. The inelastic energy transferred to the lattice during inelastic collisions is dissipated as heat.

The total energy loss is then given by

$$
\left(\frac{\mathrm{dE}}{\mathrm{dx}}\right)_{\mathrm{tot}}=\left(\frac{\mathrm{dE}}{\mathrm{dx}}\right)_{\mathrm{n}}+\left(\frac{\mathrm{dE}}{\mathrm{dx}}\right)_{\mathrm{e}}
$$

which is approximately a constant for a given implantation energy.

Once $S_{e}$ and $S_{n}$ are known, the total path length, $R$, or range of the implanted ions can be determined. The total range depends on the initial incident ion energy and is given by:

$$
R=\frac{1}{N} \int_{0}^{E} \frac{d E}{S_{n}(E)+S_{e}(E)}
$$

The projected range $R_{p}$ is the projection of $R$ in the incident direction of the ion beam. The standard deviation of the projected range is $\Delta R_{p}$. Lindhard, Scharff, and Schiott $^{5}$ were the first to show theoretically that the ion range can be 
described by a Gaussian distribution. Figure 2.4 illustrates the basic range parameters for an implanted ion.

In addition to the projected range, $R_{p}$ and the standard deviation $\Delta R_{p}$, a third parameter which is important in the practical use of ion implantation is the lateral spread, $\Delta R_{p L}$. The lateral spread is the range to which the ions are scattered away from their incident direction, as shown in Figure 2.4. The lateral spread is approximately equal in magnitude to the standard deviation and is small compared with the lateral diffusion. However in modern devices the dimensions are small enough that lateral spread becomes important. In addition, when implantation through a mask takes place the lateral spread results in an effective broadening of the structure (Figure 2.5).

The ion concentration profile as a function of distance from the surface of the substrate, $x$, is given by

$$
N(x)=\frac{\phi}{\sqrt{2 \pi} \Delta R_{p}} \exp \frac{-\left(x-R_{p}\right)^{2}}{2 \Delta R_{p}{ }^{2}}
$$

This equation represents a Gaussian distribution where $\phi$ is the implant dose. Figure 2.4 shows an ion implantation profile and Table 2.2 lists the normalized ion concentration for various distances from the peak concentration. Note the ion concentration falls to $60 \%$ of its peak value at $x=R_{p}+\Delta R_{p}$. Ion profiles of this type are usually referred to as LSS profiles after Lindhard, Scharff and Schiott. 5 The maximum dopant concentration occurs at $x=R_{p}$ and is given by

$$
N_{\max }=\frac{\phi}{\sqrt{2 \pi} \Delta R_{p}} \approx \frac{0.4 \phi}{\Delta R_{p}} .
$$

These equations are valid only for implantation into an amorphous material. However, most implantations are performed with crystalline semiconductors. lons implanted into a crystalline material may travel along rows of lattice sites. This effect is called channelling, and it will be discussed in a later section. 

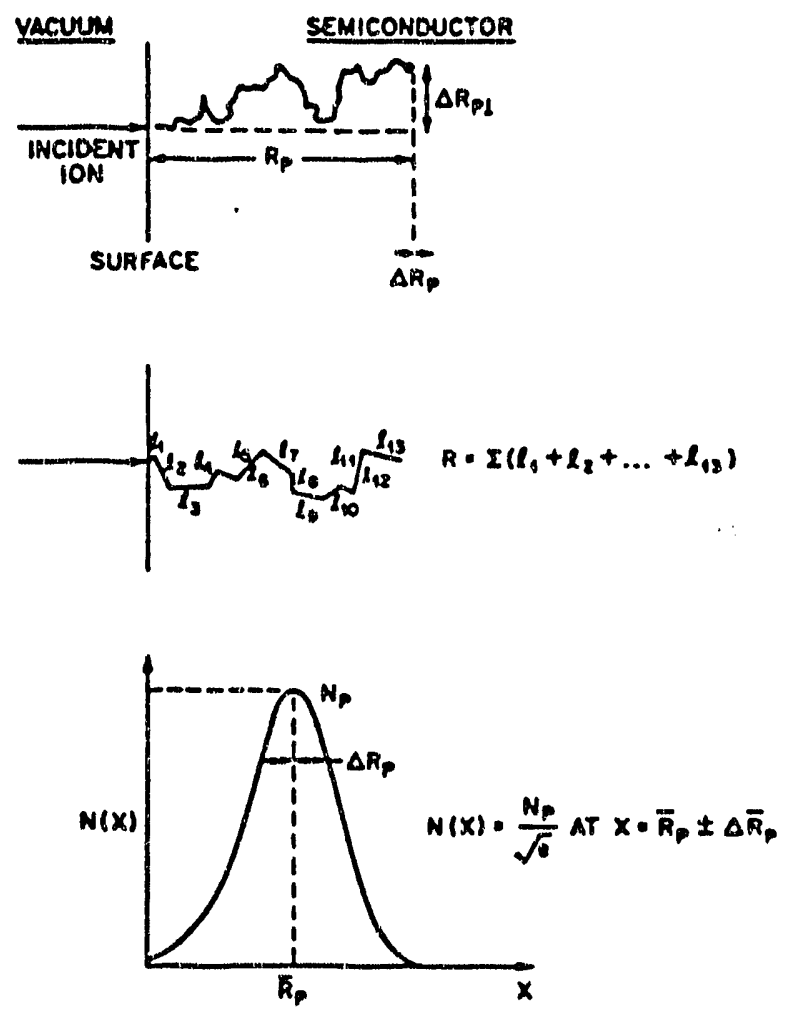

Figure 2.4. Basic range parameters for an implanted ion. (From Ref. 74) 


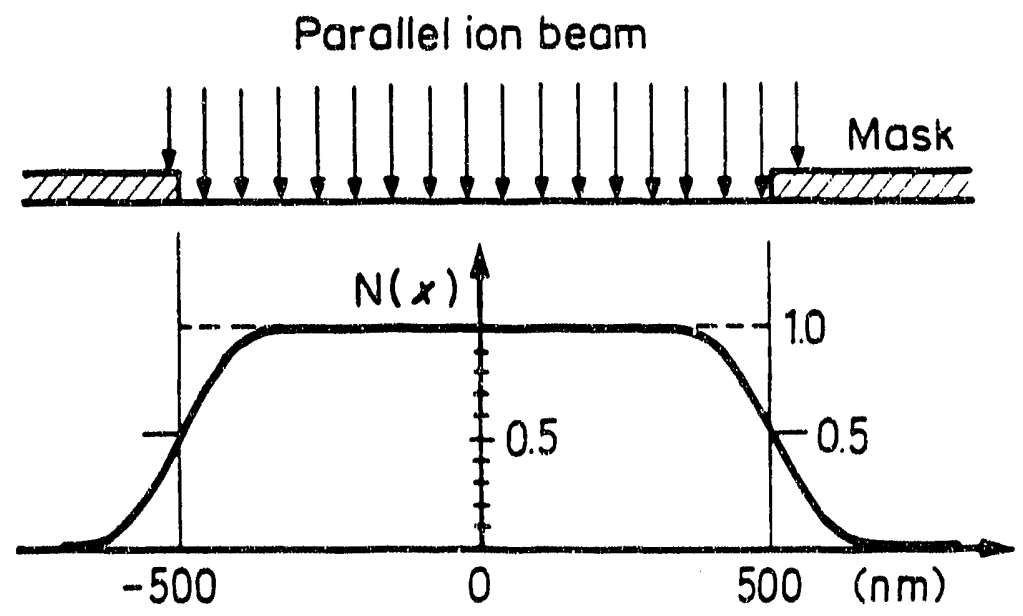

Figure 2.5. Normalized implanted ion concentration at the depth of $R_{p}$ vertical to the implantation direction after implantation through a $1 \mu \mathrm{m}$-wide mask. (From Ret. 4.)

Table 2.2. Normalized ion concentration for various distances form the peak concentration. (From Ref. 74.)

\begin{tabular}{c|c} 
Normalized Ion Conc. & Distance from Peak Conc. (Rp) \\
\hline 1.0 & 0 \\
0.5 & $\pm 1.18 \Delta R p$ \\
0.1 & $\pm 2.14 \Delta R p$ \\
0.01 & $\pm 3.04 \Delta R p$ \\
0.001 & $\pm 3.72 \Delta R p$
\end{tabular}




\subsection{Radiation Damage}

An implanted ion undergoing a nuclear collision with a substrate atom can displace the substrate atom from its lattice position. The recoiling atom can then displace other lattice atoms leading to collision cascades. Particularly at high dose rates considerable damage can occur to the substrate since recombination of point defects (e.g. self-annealing of Frenkel pairs) does not have time to occur before more damage is created. Damage clusters begin to form and eventually overlap. An amorphous layer results when all nuclei are displaced from their lattice positions and no long range order remains.

lons which are substantially lighter than the atomic mass of the constituents of the substrate only cause a small amount of damage as shown in Figure 2.6. The light ions are initially slowed by electronic stopping which causes little damage to the lattice $\left(E_{0}>M_{1} \mathrm{keV}\right)$. At the end of their range they

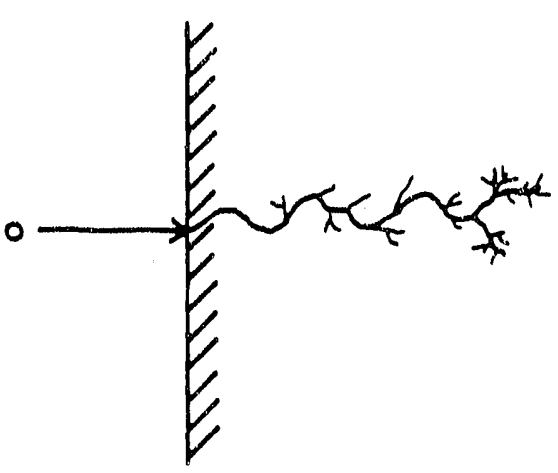

(a)

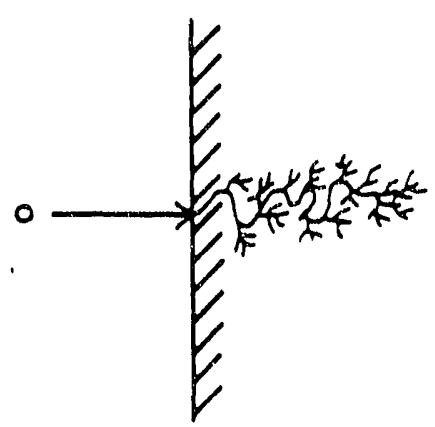

(b)

Figure 2.6. Schematic of an ion track in a solid and the associated damage for a) a light ion and b) a heavy ion. (From Ref. 4) 
are stopped by nuclear collisions. The possibility of scattering through large angles after a nuclear collision creates a particularly tortuous track for a light ion. Nearly all the damage caused by the implanted ion of small atomic weight occurs at the end of its range.

On the other hand, heavy ions create considerable damage along their entire path (Figure 2.6). They displace target atoms from the surface inwards due to a relatively higher number of nuclear collisions (as compared with light ions). Recoiling substrate nuclei can also displace other nuclei resulting in considerable lattice damage in a small volume, creating damage clusters.

For both light and heavy ions the volume of crystal in which the energy of the implanted ion is deposited is larger than the volume in which damage occurs. Near the end of its path in the crystal, the ion no longer has enough energy to displace substrate atoms. Therefore, the range of the damage does not coincide precisely with the ion profile in the solid with the peak of the damage profile occurring at $0.7-0.8 R_{p}$. (Figure 2.7)

The simplest defects created during implantation are Frenkel pairs, consisting of a vacancy and an interstitial. More complex defects can also be formed: di-vacancies, tri-vacancies, clusters of vacancies, and clusters of interstitials. Vacancies and interstitials can have various charge states and may form complexes with impurities thus affecting diffusion and electrical characteristics of the implanted layer. Line defects will occur due to the accumulation of point defects. Dislocations can grow during annealing into the undamaged part of the crystal.

The total number of atoms displaced by an incoming ion 6 is

$$
N_{d}=\frac{E_{n}}{2 E_{d}}
$$




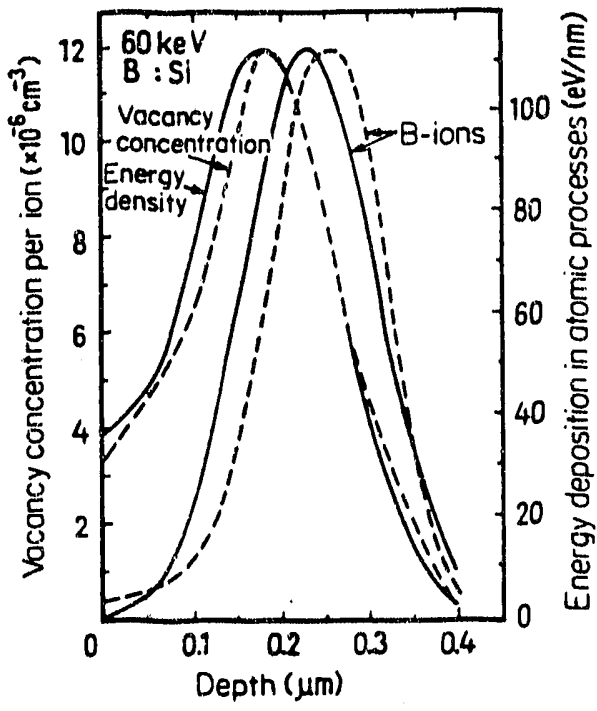

Figure 2.7. Comparison between energy distribution deposited into atomic process and Monte Carlo calculation of vacancy concentration. The ion distributions based on both theories are also given. (From Ref. 4.).

where $E_{n}$ is the total loss of energy by an incoming ion in secondary and primary nuclear collisions and $E_{d}$ is the displacement energy of a lattice at $2 \mathrm{~m}$. In semiconductors $E_{d}$ is typically about $20 \mathrm{eV}$. Assuming all atoms in the target must be displaced to form an amorphous layer a critical dose can be determined.

$$
\phi_{\mathrm{AM}}=\frac{2 \mathrm{E}_{\mathrm{d}} \mathrm{N}}{\left(\frac{\mathrm{dE}}{\mathrm{dx}}\right)_{\mathrm{n}}}
$$

Using the above equation, Si implanted into GaAs at an energy of $40 \mathrm{keV}$ will create an amorphous layer at a dose above $10^{14} \mathrm{~cm}^{-2}$.

Equation [2.26] only gives a lower limit of the critical dose due to the effects of self-annealing and the recombination of vacancies and interstitials. 
The effective critical dose strongly depends on the substrate temperature and the dose rate. In general, increasing the temperature increases the critical dose since self annealing occurs at a higher rate. Increasing the dose rate decreases the critical dose since damaged areas will accumulate and be unable to self-anneal. However, very high dose rates will cause heating of the substrate and increase dynamic annealing. Experimentally determined critical doses for creating an amorphous layer in GaAs implanted at room temperature are approximately: $10^{15} \mathrm{~cm}^{-2}$ for carbon $7,2 \times 10^{14} \mathrm{~cm}^{-2}$ for silicon 7 and $3 X$ $10^{13} \mathrm{~cm}^{-2}$ for zinc and cadmium 8 .

The damage caused by the implanted ions will decrease the mobility of the charge carriers in the semiconductor. Deep levels created by radiation damage serve as free carrier traps and recombination centers. Thus, following implantation, most semiconductors exhibit high resistivity before annealing. This property is used for damage induced device isolation in GaAs. ${ }^{9}$

\subsection{Channeling}

Channeling occurs when ions travel along rows of lattice sites. Very few nuclear collisions occur in this case. The ions will penetrate into the substrate much deeper than if they were not channeled. The only energy losses which occur are those due to electronic losses.

Once the ion enters a channel it is "guided" or "steered" by the repelling potential of the row of atsms. If the angle of entry is below a critical angle the implanted ion will be confined to a channel (Figure 2.8). The range of the ion is then directly proportional to the velocity of the ion. The critical angle can be determined by dividing the incoming energy of the ion into $E_{\perp}$ and $E_{\|}$to the channel axis. If $E_{\perp}<V(r)$, the repelling potential of the atomic chain, the ion will remain in the channel. The critical angle in GaAs is between 10 and $10^{\circ}$ for the 


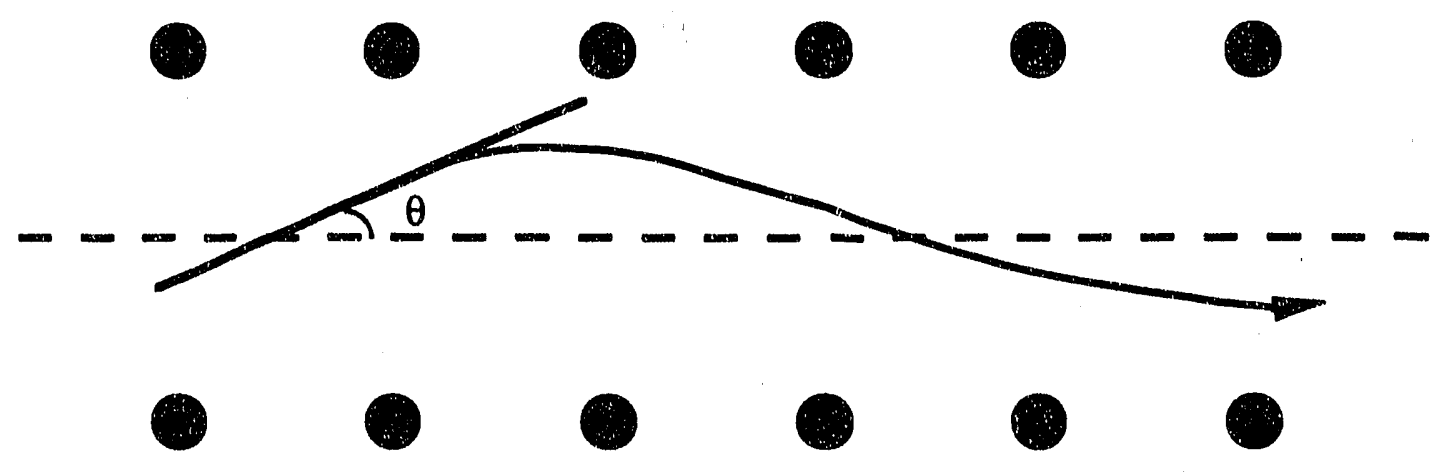

Figure 2.8. Schematic illustration of an ion path in a channel where the angle of entry is less than the critical angle.

major crystal axes and typical implant energies. The principal channelling directions in GaAs are $\langle 110\rangle,\langle 111\rangle$, and $\langle 100\rangle$.

Complete channelling is never achieved due to randomization of the beam caused by surface scattering. Ions can be channeled immediately upon entering the crystal or scattered into a channel after undergoing a few nuclear collisions as illustrated in Figure 2.9. Channeling can be minimized by orienting crystals in a nonchanneling direction. Minimizing channeling is particularly important in cases where control of the ion profile is critical for device performance. At higher doses damage created by the implanted ions will randomize the beam. Implanting through thin deposited amorphous layers will also help to randomize the beam. The only completely effective method of randomizing the beam is to pre-amorphize the material. 


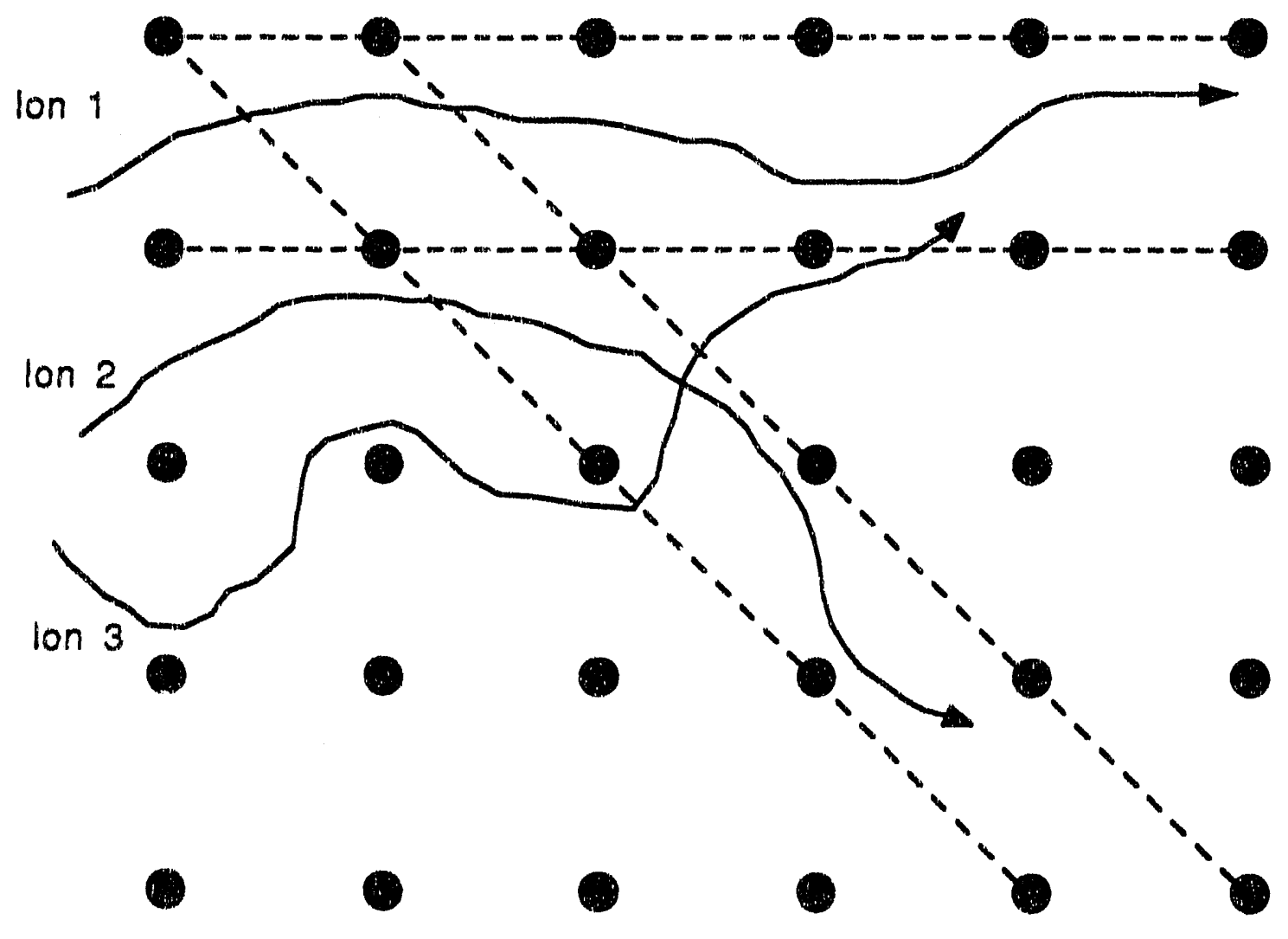

Figure 2.9. Schematic trajectories of ions through a crystal lattice. Ion 1 is channelled immediately upon entrance into crystal. Ion 2 is falls into another channel after suffering several collisions, and ion 3 is not channelled but follows a random path through the crystal. 


\section{ION IMPLANTATION IN GaAS}

\subsection{Typical Dopants}

$\mathrm{Si}$ is the most common n-type dopant in GaAs ( $\mathrm{Si}_{\mathrm{Ga}}$ ). It is easily implanted due to its low mass, allowing for greater penetration depths, and room temperature implants are readily activated. The implantation profile is essentially Gaussian and no significant diffusion occurs up to a temperature of $920^{\circ} \mathrm{C} .{ }^{10}$ Low doses $\left(<10^{13} \mathrm{~cm}^{-2}\right)$ can be thermally activated up to $80 \%$ with a rapid thermal or standard furnace anneal.11,12 For high implantation doses activation decreases due to the amphoteric nature of $\mathrm{Si}$ in GaAs. ${ }^{13}$ (Si on an As site is an acceptor.) The percentage of implanted Si atoms which sit on an As site increases as the free carrier concentration increases. The practical doping limit of Si implanted into GaAs is $5 \times 10^{18} \mathrm{~cm}^{-3}$ although peak carrier concentrations as high as $9 \times 10^{18} \mathrm{~cm}^{-3}$ have been attained. ${ }^{14}$

$\mathrm{Se}$, a group $\mathrm{VI}$ element, is also used as a donor ( $\mathrm{Se} A s)$. Se has a higher atomic mass than $\mathrm{Si}$ and therefore a smaller penetration depth for a given energy. A higher level of damage occurs in the substrate hence optimum activation occurs if implantation is performed at a temperature above $150^{\circ} \mathrm{C}$ and higher annealing temperatures are required. Se is not amphoteric, i.e., it is always a donor, so higher doping levels can be achieved. The peak carrier concentration obtained for Se implantation ${ }^{15}$ is $3 \times 10^{19} \mathrm{~cm}^{-3}$. Similar results are obtained for other group VI elements such as $S$ and $T e .{ }^{16}$

Implantation of p-type dopants has been more successful (i.e., activation efficiencies are higher) than implantation of donors. All group II elements (Be, $\mathrm{Mg}, \mathrm{Zn}$, and $\mathrm{Cd}$ ) exhibit high activation following implantation, and annealing temperatures can be kept lower than temperatures used in annealing following donor implantation. In general, optimum electrical characteristics are obtained by annealing between 700 and $800^{\circ} \mathrm{C}$. However the group II elements suffer 
from significant redistribution and loss of dopant to the surface during annealing. $\mathrm{Zn}$ is the most commonly implanted group 11 dopant since implantation sources are easily obtainable and high peak hole concentrations are attainable. Peak carrier concentiations near $10^{20} \mathrm{~cm}^{-3}$ have been obtained. ${ }^{17}$ Be has also received considerable attention since it is the lightest p-type dopant and has the greatest penetration depth. Mg offers a good compromise since its atomic mass lies between $\mathrm{Be}$ and $\mathrm{Zn}$. Good activation of $\mathrm{Mg}$ requires careful control of implantation parameters and annealing conditions.

\subsection{Defects Created by Implantation}

Defects created in GaAs during implantation play a much more important role in the electrical characteristics of the material than defects in silicon or germanium. In $\mathrm{Si}$ and $\mathrm{Ge}$, a 1:1 correspondence between solubility and electrical activity exists, i.e., a dopant will be electrical active if it occupies a substitutional site. However in GaAs, a compound semiconductor, a copant atom which sits substitutionally will not necessarily contribute a free carrier. For example, $\mathrm{Si}$ is an n-type dopant in $\mathrm{GaAs}$, however a portion of the implanted $\mathrm{Si}$ atoms can occupy As sites. SiAs ${ }^{-}$will act as an acceptor and compensate the $\mathrm{SiGa}^{+}$donors. In addition, point defects, such as $\mathrm{As}$ and $\mathrm{Ga}$ vacancies and interstitials, are created during the implantation and annealing process. These native defects whose energy levels lie in the band gap can compensate implanted dopants by trapping free carriers. This leads to a reduction of the electrical activity. Native defects can also form neutral complexes with the substitutional dopant atoms resulting in low electrical activity.

In GaAs, the highest free carrier concentration which can be obtained for $n$-type dopants is limited. The limit is not related to the total solubility limit of the 
specific impurities since it has been demonstrated that the total impurity concentration can be much higher than the free carrier concentration. ${ }^{18,19}$ The process which limits the free carrier concentration in GaAs is related to the mechanism of native defect induced Fermi level stabilization, a model proposed by Walukiewicz. 20 Stabilization of the Fermi energy is a direct consequence of amphoteric behavior of simple native defects which can undergo structural transformations, hence changing their electrical properties. The type of generated defects is determined by the Fermi energy position and acceptor- or donor-like defects are preferentially generated in $n$-or $p$ - type material respectively. The stabilization energy for GaAs lies between 0.6 and $0.8 \mathrm{eV}$ above the valence band as determined by Fermi level pinning at metalsemiconductor interfaces and in heavily irradiated substrates.21,22 The stabilization of the Fermi energy, $E_{F S}$, corresponds to a minimum free energy of the defect system in quasi-equilibrium with the free carrier gas. 22

The model of amphoteric native defects proposed by Walukiewicz postulates that implanted GaAs is compensated by the native defects generated during implantation. During post-implantation annealing intentionally introduced impurities are activated and damage is removed. However, the process of the damage removal is less efficient in material with high free carrier concentration. As the free carrier concentration increases the Fermi level differs significantly from the stabilization energy. Native defects which compensate the majority dopants become energetically favorable. In such a case the annealing process cannot completely eliminate residual defects which compensate the introduced donors or acceptors, limiting the maximum free carrier concentration. Introduction of more impurities will result only in a higher concentration of compensating defects and cannot lead to higher free carrier concentrations. This interaction results in lower implant activation efficiency. 
The energy determining maximum carrier concentration is related to the energy separation $\left|E_{1}-E_{F S}\right|$. To predict implant activation efficiencies and/or maximum free carrier concentrations one needs to know this energy at high temperatures. Since $E_{F S}$ in GaAs is closer to the valence band than the conduction band, $n$ type doping will be less efficient than p-type doping. Ion implantation of $n$-type dopants produces maximum practically obtainable free carrier concentrations of only $5 \times 10^{18} \mathrm{~cm}^{-3}$ however with p-type dopants hole concentrations near $10^{20}$ $\mathrm{cm}^{-3}$ can be obtained.

\subsection{Stoichiometry Effects}

Ion implantation into compound semiconductors is more complicated than implantation in elemental semiconductors due to the presence of more than one substrate element. Implanted dopants are expected to substitute for a particular constituent of the lattice. Consider C implanted into GaAs. C has been found to act as a shallow p-type dopant and therefore it must occupy an As site. Implanting $C$ results in the build-up of the As sublattice and a corresponding deficiency of the $\mathrm{Ga}$ sublattice. This deficiency is then compensated by the creation of native defects, in this case, Ga vacancies or As interstitials.

$$
C \Leftrightarrow C_{A S}+V_{G a} \quad \text { or } \quad C \Leftrightarrow C_{A s}+A s_{i} .
$$

The electrical activity of these defects may be such that they compensate the dopant atoms as described in the previous section. Also, the native defects can diffuse during annealing and form complexes with the dopants which are electrically inactive. Another possibility is for $\mathrm{C}$ to occupy $\mathrm{Ga}$ sites, however, no experimental evidence of $\mathrm{C}_{\mathrm{Ga}}$ exists.

Heckingbottom and Ambridge 23 proposed the co-implantation of a complementary species to reduce stoichiometry effects. In this case, since C 
sits on the As site, Ga or another group III element, (i.e., B, Al, or In) would need to be implanted to maintain stoichiometry during the implantation and annealing process. What is typically understood by co-implantation is implantation of a second species with the same distribution profile as the primary dopant. The second species (co-implant) is implanted either before or after the dopant implantation. The energy and dose of the co-implant is chosen such that its range and distribution matches that of the dopant species, allowing for maximum stoichiometric benefits.

Various groups have tested this theory with the more common dopants in GaAs. The n-type activation efficiency of Si has been shown to increase when co-implanted with $\mathrm{P}$ or As. ${ }^{24-28}$ The electron mobility also increased after coimplantation although optimum electrical characteristics were obtained for higher temperature anneals following co-implantation as compared with $\mathrm{Si}$ implanted alone. Similar results were obtained for Se. Co-implantation of $\mathrm{Ga}$ increased the activation and the mobility in several donor studies. $26,29,30$

Improvements were obtained in activation efficiency of group if acceptors when co-implanted with a group $V$ element (either $P$ or As). Increased electrical activity and less broadening of the implant profile due to diffusion during annealing was found for $\mathrm{Zn}+\mathrm{P}$ and $\mathrm{Be}+\mathrm{P}$ co-implants, ${ }^{31} \mathrm{Mg}+\mathrm{As}$ coimplants, 32 and $\mathrm{Zn}+$ As co-implants. 33 In all cases optimum electrical characteristics were obtained at higher annealing temperature for co-implants then for the group II element implanted alone. Hole mobilities were found to be lower in the case of co-implants.

Activation efficiency was not found to depend on the sequence of the implantations. Whether the co-implant was implanted first or the dopant was implanted first did not change the final electrical characteristics. 29, 32 
Two other issues related to stoichiometry affect the success of ion implantation in GaAs: the deviation from stoichiometry of the initial substrates and the loss of the low vapor pressure species (As) during high temperature anneals. The composition of GaAs crystals varies depending on growth conditions and melt composition. In addition, wafers from the same ingot may have different compositions. A clear and dramatic dependence of Si donor activation efficiency on melt composition of GaAs crystals was shown by Von Neida et. al. 34 The activation efficiency varied from $26 \%$ activation for samples with 47.5 atomic\% As to $91 \%$ activation for samples with 65 atomic\% As in the melt. The electron mobility was also higher in the latter case. Clearly, excess As aided in Si choosing a substitutional Ga site and therefore acting as a donor. From this result, it follows that local deviations from stoichiometry will play a role in dopant activation. Therefore, the effect of co-implantation can vary depending on the composition of the substrate. A low vapor pressure constituent such as As in GaAs is often lost during high temperature annealing. Local variations in composition induced by nonuniform heating during annealing following implantation will result in increased As loss from the hotter sections of the wafer. This issue will be dealt with in detail in a later section. Obviously, control is required in each processing step for reproducible implantation results, from the starting substrate to the annealing step.

\subsection{Implantation Damage in GaAs}

Christel and Gibbons ${ }^{35}$ used a Boltzman transport equation to calculate deviations from stoichiometry which result from ion implantation of various dopants in compound semiconductors. In all cases, an excess concentration of the heavier element existed at shallow depths while an excess concentration of th. lighter element existed at greater depths. The transition point occurs near 
the peak of the implanted ion distribution. For GaAs, the recoling of $\mathrm{Ga}$ and $\mathrm{As}$ from the near-surface region results in a net vacancy concentration in this region. The Ga and As atoms pile up deeper in the substrate producing a local net exçss. Due to the difference in $\mathrm{Ga}$ and $\mathrm{As}$ atomic masses, the $\mathrm{Ga}$ atoms will recoil slightly further and therefore the surface region is slightly As rich and the deeper region is slightly $\mathrm{Ga}$ rich. The calculated $\mathrm{Ga}$ and $\mathrm{As}$ recoil profiles are shown in Figure 3.1.

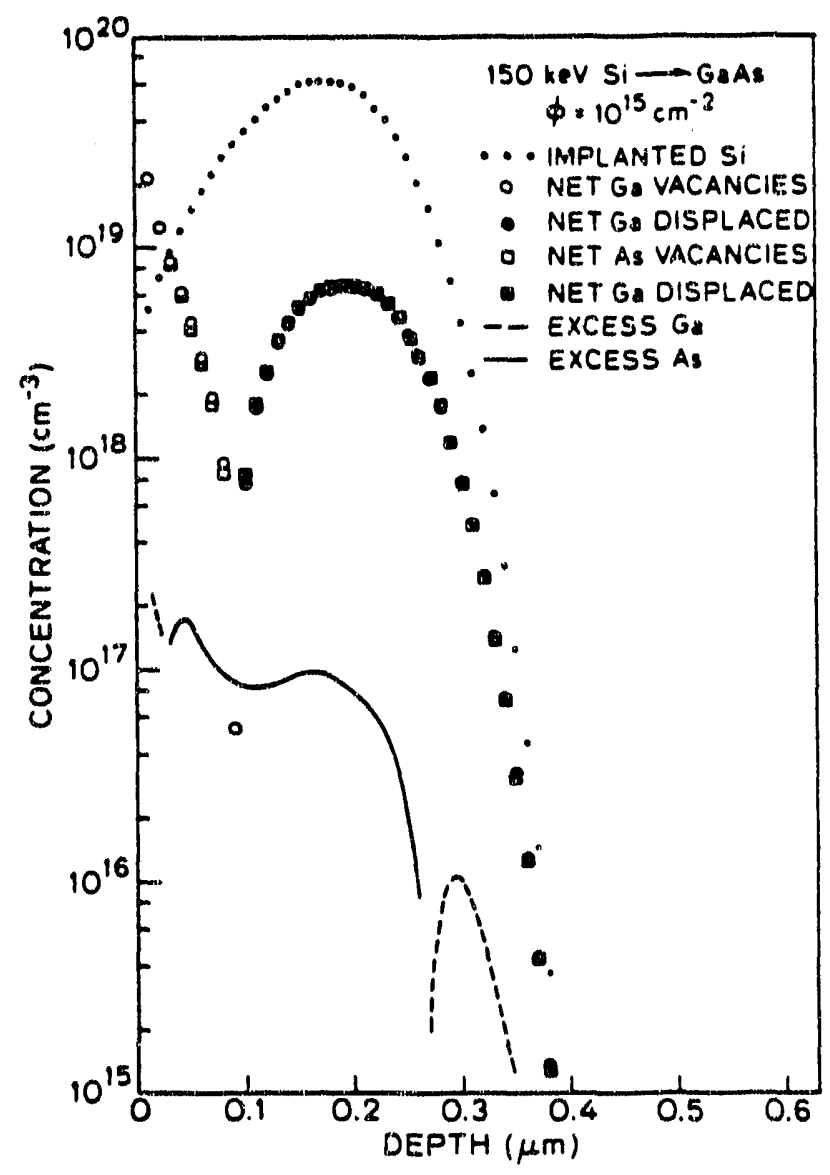

Figure 3.1. Calculated distribution of $\mathrm{Ga}$ and $\mathrm{As}$ in $\mathrm{GaAs}$ implanted with 150 $\mathrm{keV} \mathrm{Si}$ at a dose of $10^{15} \mathrm{~cm}^{-2}$. (From Ref. 35 ) 
When the substrates are annealed following implantation, proper reconstruction of the lattice is dependent on the availability of the elements of the lattice in the proper proportions near the interface between the damaged area and the recrystallized layer. Variations in the local composition result in the requirement that some elements of the compound must diffuse hundreds of Angstroms to allow for the growth of $1: 1 \mathrm{Ga}$ :As epitaxial layers. A deficiency of either component in the vicinity of the regrowth boundary will create a stoichiometric imbalance leading to the creation of native defects. The substitutional site occupied by an amphoteric dopant and its electrical activity is strongly influenced by the availability of vacancies of each type. Therefore the uneven recoil will affect the stoichiometry of the regrown layer hence affecting the electrical activity of implanted dopants.

In GaAs, damage accumulation during implantation, damage recovery, and dopant activation during annealing are extremely sensitive to implantation parameters. Self-annealing during the implantation process readily occurs in GaAs. The amount of damage and therefore the crystalline - amorphous transition in implanted GaAs has been shown to strongly depend on the temperature of implantation.36 A study of GaAs substrates implanted with 100 $\mathrm{keV} \mathrm{Si}$ ions ciemonstrated the existence of a distinct transition temperature above which the substrate did not become amorphous during implantation. This transition temperature was found to be very near room temperature. The amount of damage created in the implanted GaAs has been shown to also depend on the dose rate during implantation. 37 The dose rate also has been shown to affect the activation efficiency and the mobility of Si implanted into GaAs. ${ }^{38}$ Higher dose rates were found to decrease the activation of $\mathrm{Si}$.

In Si, solid phase epitaxy (SPE) of completely amorphous layers was shown to permit ion-implanted dopants to be incorporated onto substitutional 
sites at a temperature much lower than that required for activation of an implant that causes heavy damage but does not lead to complete amorphization of the layer. ${ }^{39}$ However, in GaAs low temperature anneals of amorphous layers do not completely remove crystal defects, 40 higher temperature anneals ( $>800^{\circ} \mathrm{C}$ ) are required to remove extended defect siructures such as residual dislocation loops. Amorphization and solid-phase epitaxy have been investigated since the channeling tail associated with implants into crystalline materials could possibly be removed. Attempts to electrically activate $n$-type impurities in preamorphized GaAs layers have been unsuccessful for Si implants ${ }^{41}$ and $\mathrm{Se}$ implants, ${ }^{42}$ apparently due to precipitation of the impurities on defect structures. However, complete electrical activity of Be was achieved when the Be was implanted following an As implant at $77 \mathrm{~K} .43$ Diffusion of the $\mathrm{Be}$ was also suppressed resulting in a very abrupt beryllium-implanted layer. 


\section{THERMAL ANNEALING}

\subsection{General Requirements}

Thermal annealing is required following implantation to repair the disorder in the crystal and to activate the implanted ions by causing their shortrange diffusion to a lattice position. In general the temperature required for annealing is high enough to cause surface degradation due to loss of arsenic. Therefore, the surface must be protected in some way during annealing.

\subsection{Damage Removal}

The damage caused by implantation consists of amorphous layers or extended crystalline defects (dislocation loops and stacking faults). The stages of damage removal in GaAs are:44

1) $150-200^{\circ} \mathrm{C}$ : The amorphous layers recrystallize epitaxially however the layers are highly defective containing twins, stacking faults and other extended defects.

2) $400-500^{\circ} \mathrm{C}$ : Extended defects begin to disappear leaving behind a high density of dislocation loops.

3) $>700^{\circ} \mathrm{C}$ : The dislocation loops grow and annihilate.

4) $>750^{\circ} \mathrm{C}$ : The remaining point defect clusters anneal out.

Above $600^{\circ} \mathrm{C}$ the surface must be protected to prevent surface degradation. Excess diffusion of dopants (especially group II acceptors) occurs above 700 ${ }^{\circ} \mathrm{C}$.

\subsection{Protection of Surface}

To preserve the integrity of the surface, the loss of As must be prevented. Various methods are commonly employed: 
1) Encapsulating the surface with a dielectric. The first requirement for an encapsulating layer is that it does not react with the host. Also, thermal expansion coefficients should be as closely matched as possible to prevent stress during annealing. The most commonly used encapsulant are $\mathrm{SiO}_{2}$ or $\mathrm{Si}_{3} \mathrm{~N}_{4} . \mathrm{Si}_{3} \mathrm{~N}_{4}$ often suffers from cracking and pealing. $\mathrm{SiO}_{2}$ has a significantly different coefficient of thermal expansion and therefore near surface strains result which can lead to significant enhancement of diffusion in the implanted layers. Both $\mathrm{Ga}$ and $\mathrm{As}$ atoms have been detected in $\mathrm{SiO}_{\mathrm{x}}$ and $\mathrm{SiN}_{\mathrm{y}}$ films after high temperature $\left(>800^{\circ} \mathrm{C}\right)$ heat treatment. The concentration of $\mathrm{Ga}$ atoms prevails over that of As atoms in $\mathrm{SiO}_{x} / \mathrm{GaAs}$ systems and vice versa in $\mathrm{SiN}_{\mathrm{y}} / \mathrm{GaAs}$ systems after heat treatment. 45

2) Group V (As) overpressure. Considerable safety hazards exist in the use of As gas and the purity of the gases is unreliable.

3) Proximity method. The implanted wafer is laid face to face with another uncapped GaAs wafer resulting in an As overpressure between the two surfaces. During placement in the furnace the two wafers can move resulting in microscratches. Any dust or contamination on either wafer will be baked on both wafers.

\subsection{Rapid Thermal Annealing}

Activation of implanted dopants takes place within the first 10 seconds at elevated temperature. Any time spent at high temperature beyond that required to activate the dopants will broaden the implanted profile due to diffusion and degrade the surface. Therefore, rapid thermal annealers have been developed which operate on the time scale pf 1 to 100 seconds. In general, the optimum annealing conditions are a higher temperature and a shorter duration than conventional furnace annealing. For example, a furnace anneal to activate a 
common implant would be performed at $800-850{ }^{\circ} \mathrm{C}$ for 20 minutes. A RTA anneal to activate the same implant mosuld occur at $900 \cdot 950{ }^{\circ} \mathrm{C}$ for 5 to 10 seconds. 


\section{CARBON IN GaAs}

\subsection{Properties of Carbon in GaAs.}

Carbon is a group IV element and can in principle occupy either the Ga site and act as a donor or the As site and act as an acceptor. However, no direct evidence for $\mathrm{C}$ on the $\mathrm{Ga}$ site exists. In contrast $\mathrm{Si}$, also a group IV element, prefers the Ga site but will also sit substitutionally on an As site at high doping levels. These differences can be explained by the different electronegativities of the two elements. $C$ is highly electronegative (2.5) and with only two $2 p$ electrons prefers more electrons. $C$ very reluctantly gives up an electron and therefore much prefers the As site. Carbon has one less valence electron than arsenic so it acts as an acceptor when occupying an As site:

$$
C_{A s} \Leftrightarrow C_{A s^{-}}+h^{\prime}
$$

The acceptor level due to $C_{A s}$ resides in the band gap 27 meV above the maximum of the valence band. 46

Carbon was first detected in GaAs by Newman 47 who showed the excitation of the lucal vibrational mode associated with $C_{A s}$ occurred at 582 $\mathrm{cm}^{-1}$ at $4 \mathrm{~K}$. Carbon is a residual impurity in liquid encapsulated Czochralski grown GaAs crystals. Semi-insulating crystals typically exhibit a background $C$ concentration of between $5 \times 10^{14} \mathrm{~cm}^{-3}$ and $1 \times 10^{16} \mathrm{~cm}^{-3}$.

No direct evidence of substitutional $\mathrm{C}$ on a $\mathrm{Ga}$ site has been found to date. However, Woodhouse et. al. 48 have detected an L.VM spectroscopy line at $563 \mathrm{~cm}^{-1}$ (at $4 \mathrm{~K}$ ) in heavily $\mathrm{C}$-doped layers grown by metal organic molecular beam epitaxy (MOMBE). They postulated that this line could be the local vibrational mode associated with $\mathrm{C}_{\mathrm{Ga}}$.

Carbon is a particularly attractive acceptor in GaAs since its diffusion coefficient is several oroiers of magnitude lower than that of group II acceptors 
such as $\mathrm{Be}, \mathrm{Mg}$, or $\mathrm{Zn}$. For $\mathrm{Zn}, \mathrm{D}_{\mathrm{Zn}}=6 \times 10^{-14} \mathrm{~cm}^{2} / \mathrm{s}$ at $800^{\circ} \mathrm{C}$ and for $\mathrm{Be}, \mathrm{D}_{\mathrm{Be}}$ $=1 \times 10^{-15} \mathrm{~cm}^{2} / \mathrm{s}$ at $8000^{\circ} \mathrm{C} .49,50$ It is not possible to attain abrupt doping profiles with group II acceptors, due to diffusion of the implanted ion during annealing. The graded dopant profiles which result will lead to the degradation of electrical characteristics particularly in heterojunction bipolar transistors (HBTs) which require a thin, heavily doped p-type base layer. 51

Cunningham, et. al. 52 conducted a detailed study of $C$ doping and diffusion under various conditions to determine the effects of background doping, surface encapsulation and $A s_{A}$ overpressure. Spikes of $C$ were grown by metal Jrganic chemical vapor deposition (MOCVD) in the center of undoped, Se-doped $\left(\mathrm{n}^{+}\right)$, and $\mathrm{Mg}$-doped $\left(\mathrm{p}^{+}\right)$layers. Samples were annealed at $825^{\circ} \mathrm{C}$ for 24 hours. Two different As overpressures were used and some samples were encapsulated with $\mathrm{Si}_{3} \mathrm{~N}_{4}$. The As overpressure and the encapsulate were found to have no effect on the carbon diffusion. The highest diffusion coefficient (at $800^{\circ} \mathrm{C}$ ) was found to be $D=2 \times 10^{-16} \mathrm{~cm}^{2} / \mathrm{s}$ in the case of the $p^{+-d o p e d}$ layer, $D=1 \times 10^{-16} \mathrm{~cm}^{2} / \mathrm{s}$ for undoped GaAs and little or no diffusion was found in the $\mathrm{n}^{+}$doped layers. Other groups have found similar diffusion coefficients for $\mathrm{C}$-doped samples grown by various techniques. 53,54 These diffusion coefficients are significantly lower than those for Group II acceptors.

Carbon has also been generating renewed interest as an acceptor in GaAs because of recent successes in growing epitaxial layers with ultra-high carbon doping. Layers of GaAs doped with $\mathrm{C}$ with carrier concentrations exceeding $10^{20} \mathrm{~cm}^{-3}$ have been attained in growth by metal organic molecular beam epitaxy (MOMBE)53,55,56 and metal organic vapor phase epitaxy (MOVPE). 57,58 Renewed interest in C-doping of GaAs has led to investigation of ion implantation of $\mathrm{C}$. 


\subsection{Ion Implantation of $\mathrm{C}$ in GaAs}

Initial attempts at implantation of $C$ in GaAs yielded poor results. Less than $2 \%$ activation was found in substrates implanted with doses of $\mathrm{C}$ greater than $10^{13} \mathrm{~cm}^{-2}$, although at lower doses, higher activation was achieved. Sansbury and Gibbons 59 attained less than $2 \%$ activation efficiency for $\mathrm{C}$ doses of $4 \times 10^{13} \mathrm{~cm}^{-2}$ and $4 \times 10^{14} \mathrm{~cm}^{-2}$ after annealing at $700{ }^{\circ} \mathrm{C}$, and Harris 60 found similar results for a $\mathrm{C}$ dose of $2 \times 10^{14} \mathrm{~cm}^{-2}$ and an $800{ }^{\circ} \mathrm{C}$ anneal. Shin61 achieved a doping efficiency of $50 \%$ with a C dose of $1013 \mathrm{~cm}^{-2}$ after annealing at $900{ }^{\circ} \mathrm{C}$. Implantation of a dose of $10^{14} \mathrm{~cm}^{-2}$ yielded $12 \%$ activation corresponding to a bulk concentration of around $4 \times 10^{17} \mathrm{~cm}^{-3}$ in a study conducted by Paulson and Tam.62 These results are summarized in Appendix I.

Co-implantation of $\mathrm{Ga}$ has been shown to increase the activation efficiency of implanted $\mathrm{C}$ particularly at high doses. Shin et.al. 63 found that coimplantation of $\mathrm{Ga}$ increased the activation efficiency for $\mathrm{C}$ from $9 \%$ to $32 \%$ for a $\mathrm{C}$ dose of $10^{14} \mathrm{~cm}^{-2}$ after annealing at $900^{\circ} \mathrm{C}$. The peak bulk concentration in co-implanted samples was $2 \times 10^{18} \mathrm{~cm}^{-3}$. Ga co-implantation made a dramatic difference in the activation efficiencies obtained by Pearton et.al.64 Activation efficiencies increased from 34 to $60 \%$ for $1 \times 10^{13} \mathrm{~cm}^{-2}$ implants and from 2.5 to $43 \%$ for $5 \times 10^{14} \mathrm{~cm}^{-2}$ implants after annealing at $800 \circ \mathrm{C}$. $50 \%$ activation corresponding to a bulk doping of $5 \times 10^{16} \mathrm{~cm}^{-3}$ was also attained by Paulson and Tam62 for $\mathrm{C}$ implanted at multiple energies and annealed at $8500^{\circ} \mathrm{C}$. Results for carbon and co-implants of $\mathrm{Ga}$ are summarized in Appendix I.

\subsection{Effect of Ga Co-Implant}

The precise role of the $\mathrm{Ga}$ co-implant regarding $\mathrm{C}$ acceptor activation is unknown. Two major effects of the Ga co-implant will be described in this 
section: radiation damage and stoichiometry. First, consider the radiation damage created during implantation. $C$ has low mass (12 amu) and when implanted causes very little damage to the lattice. Radiation damage due to low mass ions occurs primarily at the end of the range of the implanted ions. ${ }^{4}$ Few vacancies are created and $C$ will have a limited number of sites where it can sit substitutionally. Heavier ions transfer more energy to the lattice atoms and will displace many lattice atoms along their entire path through the substrate. Lattice atoms recoiling from a collision with an implanted ion may have enough energy to displace other lattice atoms. Therefore, implantation of the higher atomic mass $\mathrm{Ga}$ (69 or $71 \mathrm{amu}$ ) produces significant damage to the substrate creating many vacancies and interstitials. Thus the co-implantation of $\mathrm{Ga}$ provides additional sites for the $C$ to sit substitutionally and the activation increases.

Alternatively, consider the amount of radiation damage and the resulting degree of disorder in the lattice following implantation. During thermal annealing two processes must occur to achieve high activation, the $\mathrm{C}$ must sit on an As site and a nearly perfect crystal must be recovered. The efficiency of the former process may depend on the degree of disorder in the lattice. C doping during epitaxial growth has been highly successful (as mentioned previously) indicating a natural tendency for $C$ to sit on an As site. The solid phase epitaxy (during thermal annealing) of an amorphous layer created more closely resembles epitaxial growth of GaAs than the annealing of localized damage caused by implantation. Therefore, we expect $C_{A S}$ will form more easily in highly damaged layers. Again, implantation of $\mathrm{Ga}$ atoms creates more damage in the substrate. If the damaged areas overlap, an amorphous layer extending to a certain depth will form in which there is no long range order in 
the lattice. ${ }^{4}$ Therefore an amorphous layer created by $\mathrm{Ga}$ co-implantation would aid the activation of implanted carbon.

Harris 60 found the activation of $C$ (implanted at an energy of $200 \mathrm{k \theta V}$ and dose of $2 \times 10^{14} \mathrm{~cm}^{-2}$ ) increased from $1 \%$ to $8 \%$ when implants were performed at $77 \mathrm{~K}$ and created an amorphous layer in the substrate. C implanted alone in InGaAs and AllnAs does not produce any measurable electrical activity; however $\mathrm{Ar}$ co-implantation resulted in $11 \%$ activation of $\mathrm{C}$ implanted at a dose of $5 \times 10^{14} \mathrm{~cm}^{-2}$ and energy of $60 \mathrm{keV} .66$ A plausible conclusion from these preliminary results is that additional damage is required to provide substitutional sites for $C$ within the GaAs lattice.

The other major effect of the co-implanted $G a$ is to affect stoichiometry of the implanted layer. As described earlier, maintaining stoichiometry during implantation and annealing in GaAs should increase the activation of $\mathrm{C}$. The role of the co-implanted $\mathrm{Ga}$ is to build up the $\mathrm{Ga}$ sublattice and therefore enhance the probability of $C$ finding an As site and to inhibit the formation of native defects, i.e., Ga vacancies or As interstitials which may compensate the $C$ acceptors.. The composition of the crystal can also be affected by uneven recoil of the $\mathrm{Ga}$ and $\mathrm{As}$, leading to local deviations from stoichiometry, and loss of As from the surface during annealing. 


\section{EXPERIMENTAL}

In an attempt to separate the major effects enhancing carbon activation, radiation damage and stoichiometry, the following elements were co-implanted: $B, N, A l, P, A r, G a, A s, K r$. To illustrate the chemical nature of the chosen coimplant species a portion of the periodic table is shown in Figure 6.1. The group III elements: B, Al, and Ga, should help restore the stoichiometry during the implantation and annealing procedures by building up the $\mathrm{Ga}$ sublattice while N, P and As co-implants should lead to even larger deviations from stoichiometry. The inert gasses: $A r$ and $K r$, should not produce any stoichiometric effect. The atomic masses of the co-implanted elements range from $11 \mathrm{amu}(\mathrm{B})$ to $84 \mathrm{amu}(\mathrm{Kr})$.

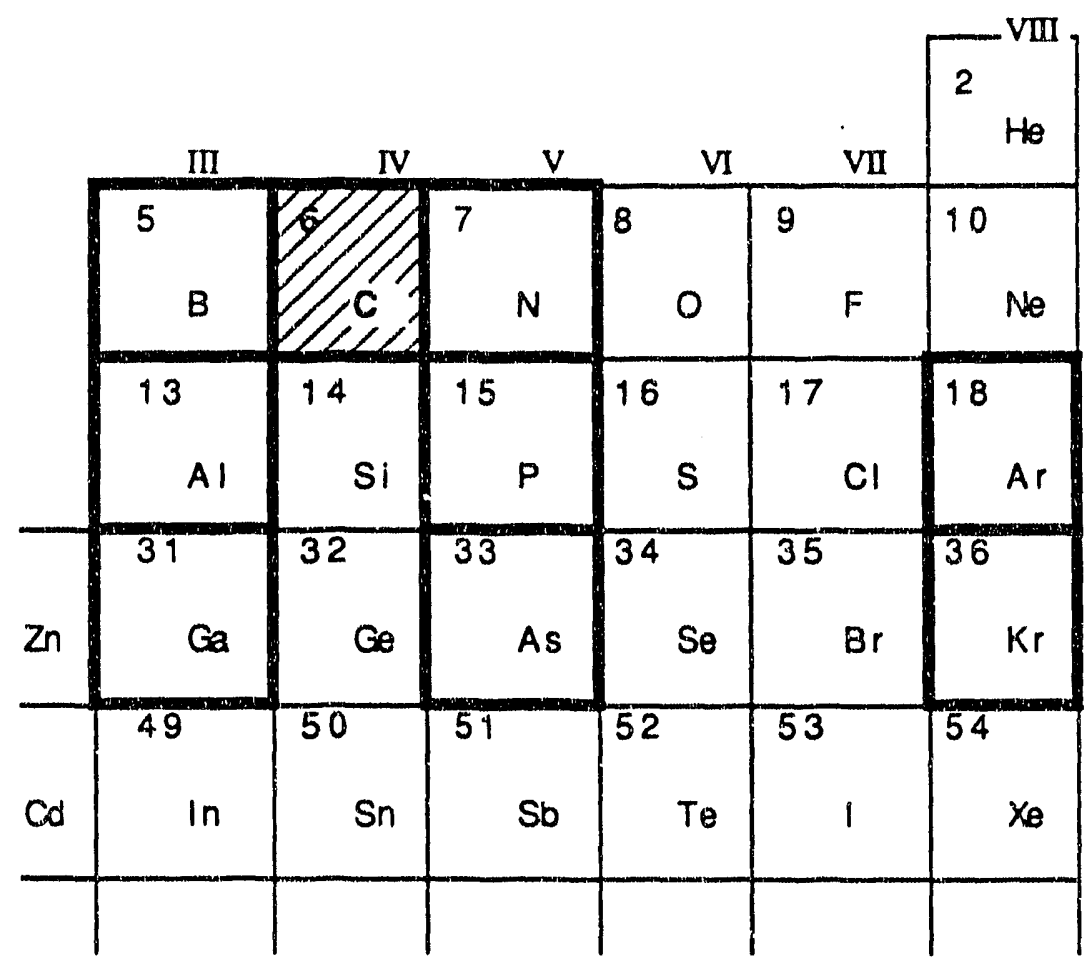

Figure 6.1. A portion of the periodic table indicating the elements implanted in this study. 
The GaAs substrates used for implantation were semi-insulating (100) Czochralski grown wafers from the M/A-Com Advanced Semiconductor Division. Before implantation the substrates were boiled in trichloroethane (TCA) for 1 minute, rinsed in room temperature TCA for 30 seconds, rinsed in methanol and dried with flowing $\mathrm{N}_{2}$. The substrates were then etched in concentrated $\mathrm{HCl}$ for 1 minute, rinsed in de-ionized water and dried in flowing $\mathrm{N}_{2}$.

The samples were mounted on an aluminum plate with wax. A small amount of silver paint was placed in the corner to maintain electrical contact between the aluminum plate and the sample. Singly ionized $C$ was implanted with an energy of $40 \mathrm{keV}$ at a dose of $5 \times 10^{14} \mathrm{~cm}^{-2}$, with the wafers tilted 70 away from the (100) direction to prevent channeling. The co-implant species was implanted following the $\mathrm{C}$ implantation (post-implantation), at a dose and energy chosen so that the profile of the co-implant matched the $\mathrm{C}$ profile according to LSS theory. The calculated profile of all implants used in this study are presented in Appendix II Energy and doses for the co-implants are given in Table 6.1. Samples ware held at room temperature during implantation.

Following implantation the samples were annealed in a Heatpulse 210 rapid thermal annealer (RTA) at $800^{\circ} \mathrm{C}$ for $10 \mathrm{~s}$ or $950^{\circ} \mathrm{C}$ for $10 \mathrm{~s}$ in flowing forming gas $\left(90 \% \mathrm{~N}_{2} / 10 \% \mathrm{H}_{2}\right)$ using the proximity method. 67 The samples were placed with the implanted side up on the Si wafer in the RTA and another piece of clean GaAs was placed on top to protect the surface. This method creates an As overpressure between the two pieces inhibiting the loss of As from the surface of the sample. 
Table 6.1. Implantation parameters for elements implanted in this study.

\begin{tabular}{cccc}
\hline Implant & $\begin{array}{c}\text { Atomic Mass } \\
\text { (amu) }\end{array}$ & $\begin{array}{c}\text { Energy } \\
\text { (keV) }\end{array}$ & $\begin{array}{c}\text { Dose } \\
\left(10^{14} \mathrm{~cm}^{-2}\right)\end{array}$ \\
\hline $\mathrm{C}$ & 12 & 40 & 5 \\
$\mathrm{~B}$ & 11 & 30 & 6 \\
$\mathrm{~N}$ & 14 & 40 & 5 \\
$\mathrm{Al}$ & 27 & 80 & 6 \\
$\mathrm{P}$ & 31 & 90 & 6 \\
$\mathrm{Ar}$ & 40 & 115 & 5 \\
$\mathrm{Ga}$ & 69 & 180 & 5 \\
$\mathrm{As}$ & 75 & 220 & 4 \\
$\mathrm{Kr}$ & 84 & 250 & 4 \\
\hline
\end{tabular}

The Heatpulse 210 RTA system consists of an annealing furnace and a microcontroller. The annealing furnace includes 13 high-intensity tungstenhalogen lamps, which are arranged in upper ( 6 bulbs) and lower ( 7 bulbs) banks and housed in water-cooled, reflective walls. A quartz annealing tube is positioned between the banks, and is hermetically sealed to the door with an 0 ring. A flat piece of quartz attached to the door holds the wafer and allows the sample loading into the isolated annealing chamber. The visible light from the continuous-wave lamps passes through the quartz annealing tube and wafer tray and is absorbed by the sample. Each of the thirteen bulbs produces $1.5 \mathrm{~kW}$ and at $100 \%$ intensity the computer limits the input power to $18 \mathrm{~kW}$. For accurate control and monitoring of the sample temperature, a thermocouple located inside the annealing chamber is hooked via a feedback loop to the controller. The thermocouple is attached to a 4 inch Si wafer and the sample is placed on the top. The thermocouple must be attached to a test wafer since the 
furnace is a cold-wall system, where only the sample and the filaments reach an elevated temperature.

The thermal cycle of the annealer consists of 5 parts: 1) the intensity of the lamps is set at $40 \%$ until the temperature reaches $4000^{\circ} \mathrm{C}, 2$ ) the RTA reprograms while holding the temperature at $400^{\circ} \mathrm{C}, 3$ ) the intensity of the lamps is increased to $80 \%$ and the temperature climbs to the chosen value, 4) the final temperature is held for the prescribed amount of time, and 5) the lamps are shut off and the temperature rapidly decreases. Heating rate is typically 60 $80^{\circ} \mathrm{C} / \mathrm{sec}$ and cooling rate from $950^{\circ} \mathrm{C}$ or $800^{\circ} \mathrm{C}$ to $600^{\circ} \mathrm{C}$ is approximately $40^{\circ} \mathrm{C} / \mathrm{sec}$. A typical heating cycle for $950^{\circ} \mathrm{C}, 10 \mathrm{sec}$ anneal and a $800^{\circ} \mathrm{C}, 10$ sec anneal is shown in Figure 6.2.

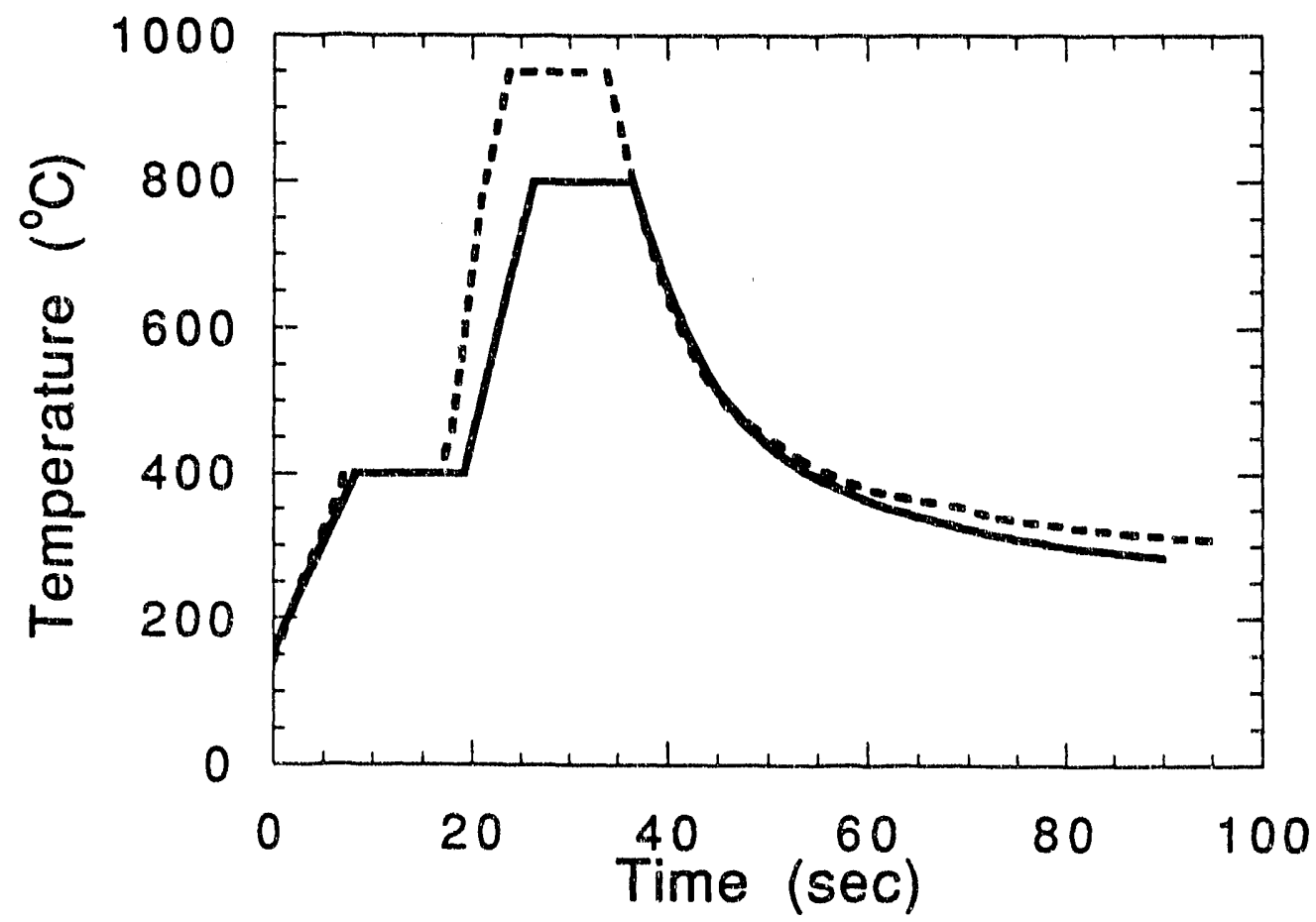

Figure 6.2. Typical heating cycles in the rapid thermal annealer used in this study. 
In the rapid thermal annealing system, the actual temperature of the sample is difficult to measure. The sample's temperature depends on absorbed light not the incident light. Therefore, the accuracy of the controlling thermocouple which is imbedded in the 4 inch Si wafer was tested. Two grooves approximately .006 inches deep were diced into a $1 \mathrm{~cm}^{2}$ piece of semi-insulating GaAs forming a cross. One groove was approximately .006 inches wide and the other 015 inches wide. A type K, bare wire thermocouple with .005 inch wires was cemented into the grooves with Savereisen and allowed to dry. The test piece was then placed in the thermocouple and another piece of semi-insulating GaAs was placed on top. Results of the temperature calibration experiment are shown in Figure 6.3. The temperature measured by the test piece did not vany more than $3.5 \%$ from the desired control temperature for any temperature above $600^{\circ} \mathrm{C}$.

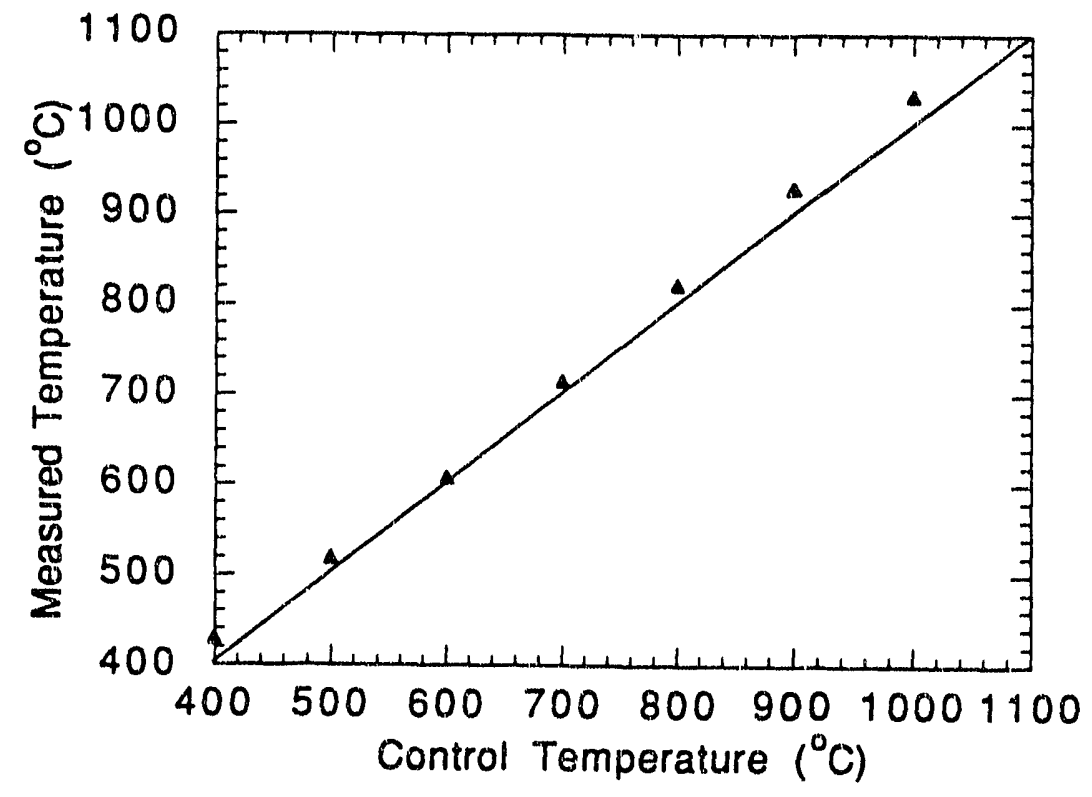

Figure 6.3. Control temperature versus measured temperature in RTA. 
Point contacts were made on the corners of $1 \mathrm{~cm}^{2}$ samples by alloying In: $\mathrm{Zn}$ contacts at $250^{\circ} \mathrm{C}$ for 10 minutes in flowing $\mathrm{N}_{2}$. Copper wires with indium coated tips were then pressed onto the contacts. Carrier concentration, mobility and resistivity were determined by van der Pauw geometry68 Hall effect measurements. All measurements were taken at a magnetic field of $3 \mathrm{kG}$. The current across the contacts was chosen so that voltages measured between 5 and $50 \mathrm{mV}$.

Rutherford backscattering spectra were taken along the $\langle 110\rangle,\langle 111\rangle$, and random directions for a set of samples. The beam consisted of $1.8 \mathrm{MeV}$ $\mathrm{He}^{+}$ions. These experiments were used to measure the amount of damage due to implantation and the subsequent repair of the damage following annealing. 


\section{RESULTS}

\subsection{Electrical Characteristics}

The complete set of Hall data from all experiments is presented in Appendix 1 . The results are summarized in the figures presented in this section. The free carrier concentrations in the bulk were determined assuming a uniformly doped layer of $100 \mathrm{~nm}$. However, the layer is not uniformly doped, rather the distribution of $\mathrm{C}$ atoms is Gaussian. Therefore, the free carrier concentrations are not entirely accurate since the distribution of the implanted C is not uniform. Sheet carrier concentration is a more appropriate measure for the implanted layer. Figure 7.1 shows the activation efficiency as a function of co-implant and anneal temperature. The activation efficiency is determined by the ratio of sheet carrier concentration to ion implant dose. Several trends are noticeable. For the co-implant species of column III (B, AI, Ga), activation increases with increasing atomic weight. This trend is also found for the coimplants from column $V(N, P, A s)$ and from the two inert gasses ( $\mathrm{Ar}, \mathrm{Kr}$ ). However for co-implants with similar atomic weights, (i.e., those from the same row of the periodic table) highest activation is found for the group III co-implant followed by the inert gas and then the group V. The lightest co-implants used $(B, N)$ have very little effect on the electrical properties. The effect of both increasing atomic mass and the chemical nature of the co-implant are shown in Figure 7.2, a plot of sheet hole concentration as a function of atomic mass.

The highest free hole concentration was attained in the case of the $C$ and Ga implant, annealed at $950^{\circ} \mathrm{C}$ for 10 seconds. The sheet carrier concentration was measured to be $3.4 \times 10^{14} \mathrm{~cm}^{-2}$, corresponding to a activation of $68 \%$. Assuming a doped layer thickness of $100 \mathrm{~nm}$, this corresponds to a bulk doping of $3.4 \times 10^{19} \mathrm{~cm}^{-3}$. To my knowledge, these are 
the highest implant activation and free carrier concentration ever found for the implantation of $C$ in GaAs.

The electrical characteristics of samples annealed at the higher temperature $\left(950^{\circ} \mathrm{C}\right)$ were better in all cases except for $\mathrm{C}+\mathrm{N}$. Sheet carrier concentrations are higher as shown in Figure 7.2 and mobilities were also higher even for higher carrier concentration (Figures 7.3 and 7.4).

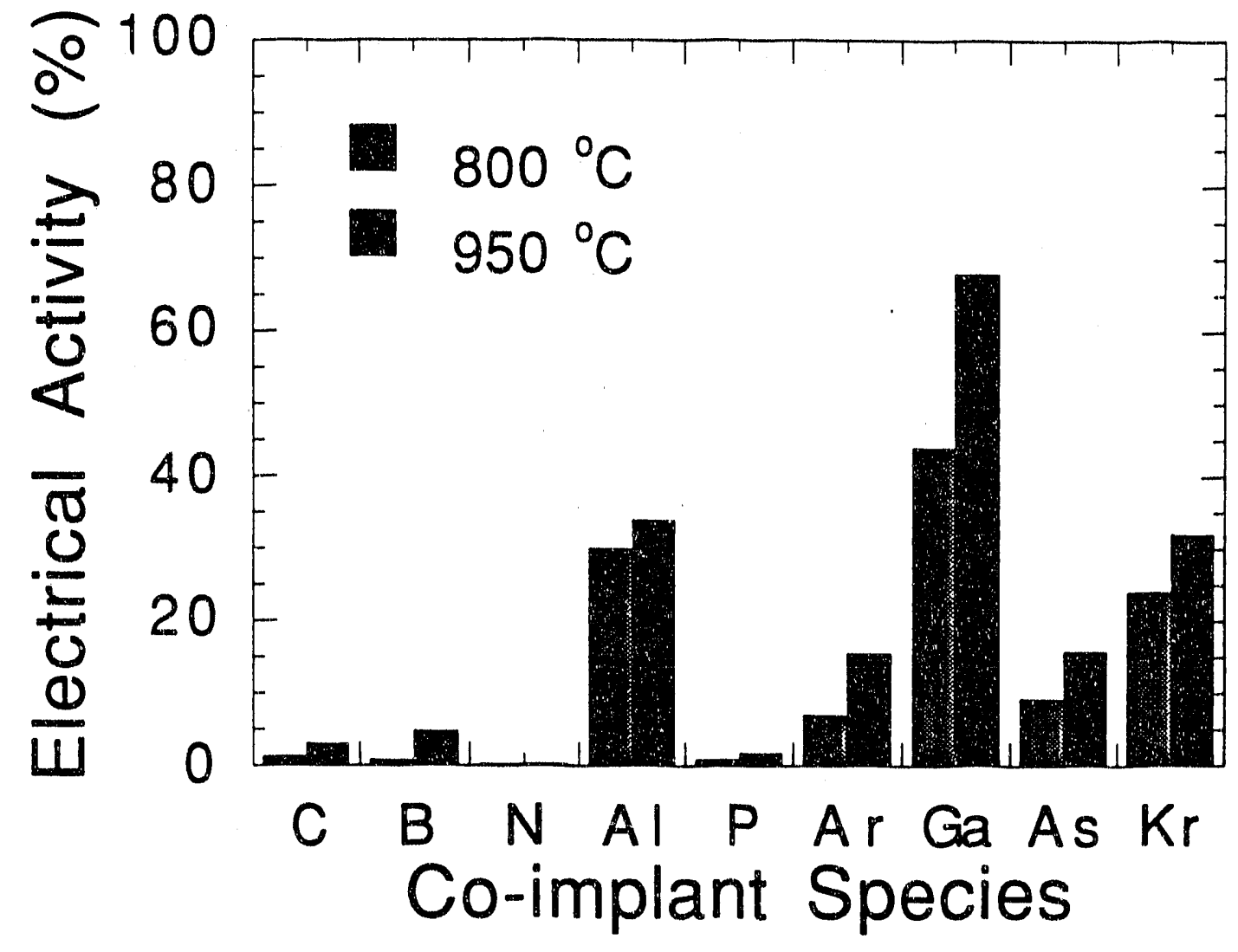

XBL $9112-2537$

Figure 7.1. Electrical activity as a function of co-implant species for samples annealed at $800^{\circ} \mathrm{C}$ and $950^{\circ} \mathrm{C}$. Electrical activity is the ratio of sheet carrier concentration to implant dose. 


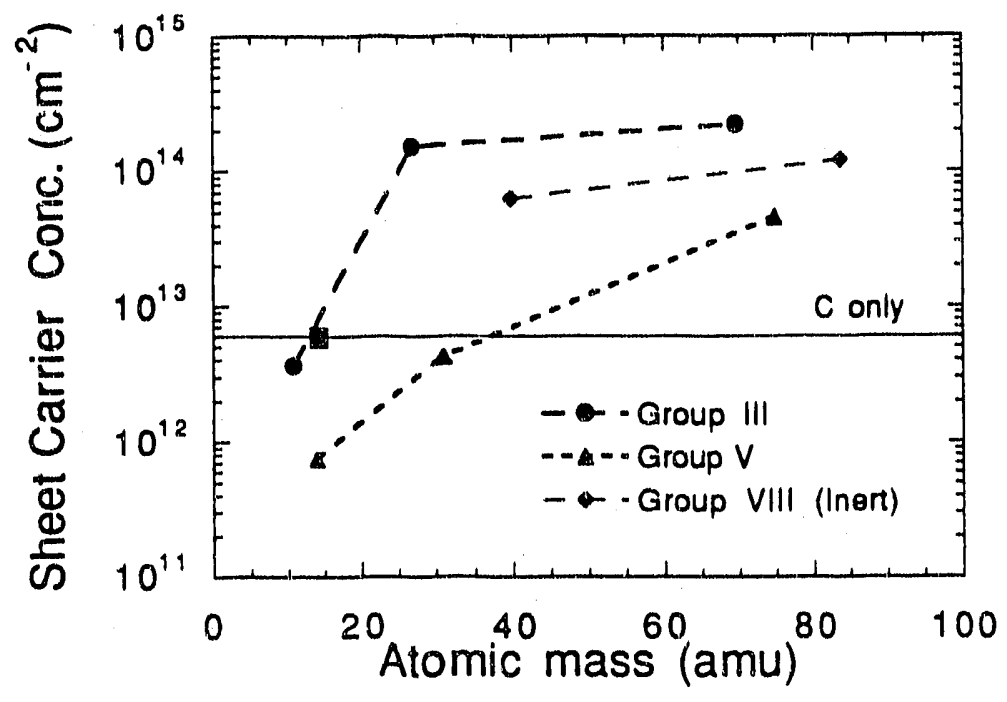

Figure 7.2 a). Sheet carrier concentration as a function of atomic mass for samples annealed at $800^{\circ} \mathrm{C}$ for 10 seconds.

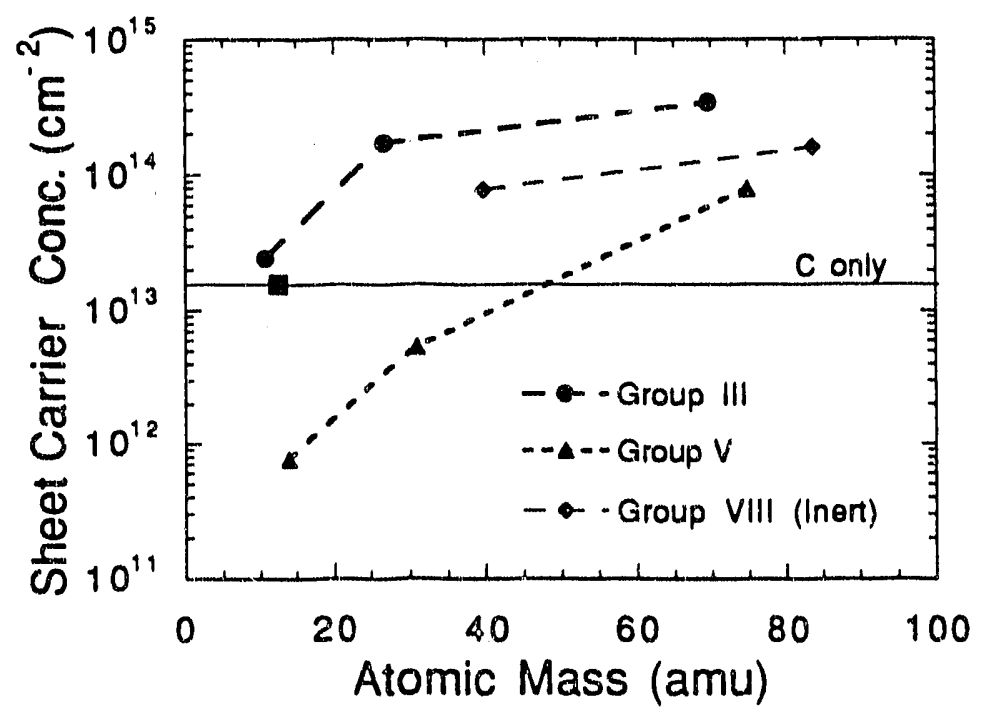

Figure $7.2 \mathrm{~b}$ ). Sheet carrier concentration as a function of atomic mass for samples annealed at $950^{\circ} \mathrm{C}$ for 10 seconds. 


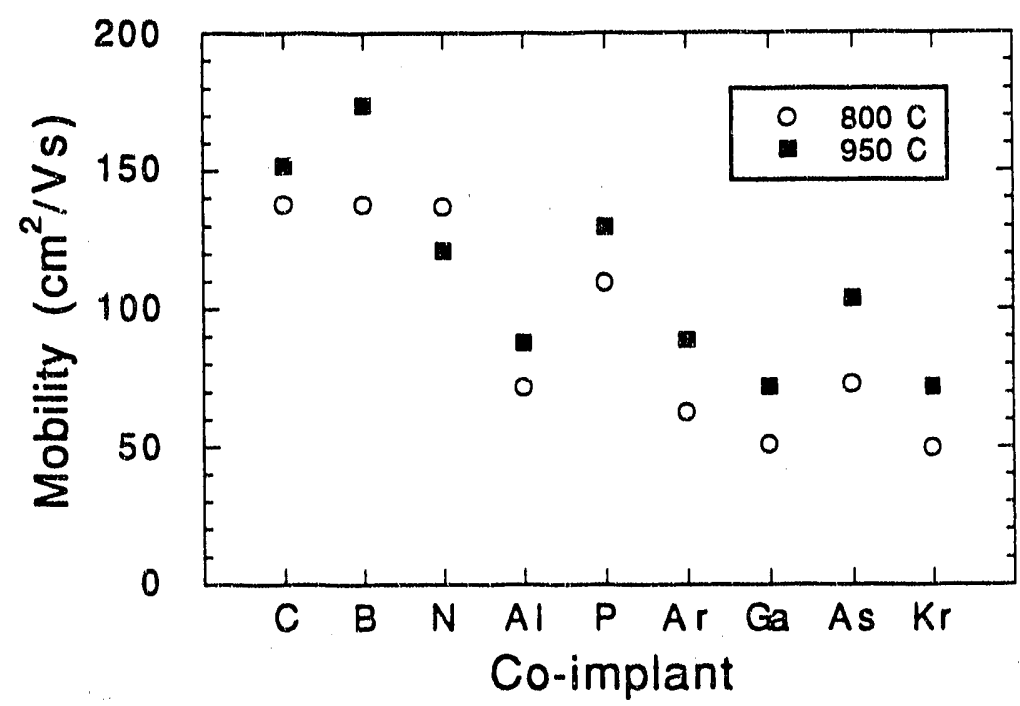

Figure 7.3. Hole mobility (Hall effect) as a function of co-implant an annealing temperature.

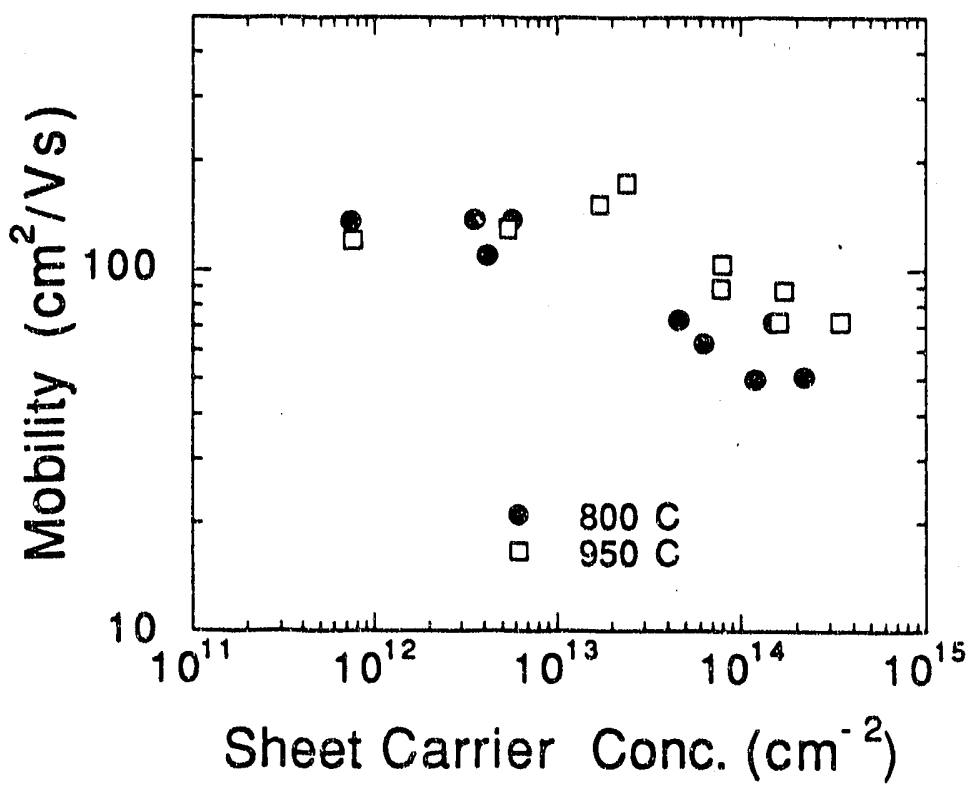

Figure 7.4. Hole mobility (Hall effect) as a function of sheet carrier concentration and annealing temperature. 


\subsection{Structural Characterization}

RBS channeling results are shown in Figure 7.5 and 7.6. $\mathrm{C}+\mathrm{Ga}$ and $\mathrm{C}+$ $\mathrm{Kr}$ implants result in an amorphous layer at the surface which is approximately $140 \mathrm{~nm}$ thick. Although As + C was not measured, it can safely be assumed that RBS spectra would look very similar. $\mathrm{Al}+\mathrm{C}$ and $\mathrm{Ar}+\mathrm{C}$ generate an amorphous layer which is about $120 \mathrm{~nm}$ thick. The $B+C$ and $C$ only implants do not cause the formation of an amorphous layer. Some damage is seen at the end of the range of the ions and the dechanneling rate is slightly higher than that in the standard (unimplanted) sample. Figure 7.7 is a magnification of the $C$ only spectrum showing the slightly damaged region at the end of the range of the ions.

Results from RBS following annealing for the $\mathrm{Ga}+\mathrm{C}$ samples are shown in Figure 7.8. The sample has recovered only slightly following the $800^{\circ} \mathrm{C}$ anneal and is nearly ideal following the $950{ }^{\circ} \mathrm{C}$ anneal. Some extended defects remain as seen by the higher dechanneling rate relative to the standard sample. Figure 7.9 compares a $\mathrm{C}+\mathrm{Ga}$ sample and a $\mathrm{C}+\mathrm{Kr}$ sample following annealing at $950^{\circ} \mathrm{C}$. Note that considerable more damage remains in the $\mathrm{C}+$ Kr sample.

SIMS results for the $\mathrm{B}+\mathrm{C}$ implant following annealing at $800^{\circ} \mathrm{C}$ are shown in Figure 7.10. The high concentration of $C$ at the surface of the sample is due to contamination from hydrocarbons present in solvents used for cleaning. Both profiles match fairly closely the profiles predicted by LSS theory. The calculated $C$ profile and the $C$ profile from SIMS measurement is shown in Figure 7.11. No significant deviations due to channeling or diffusion during the anneal are seen. 


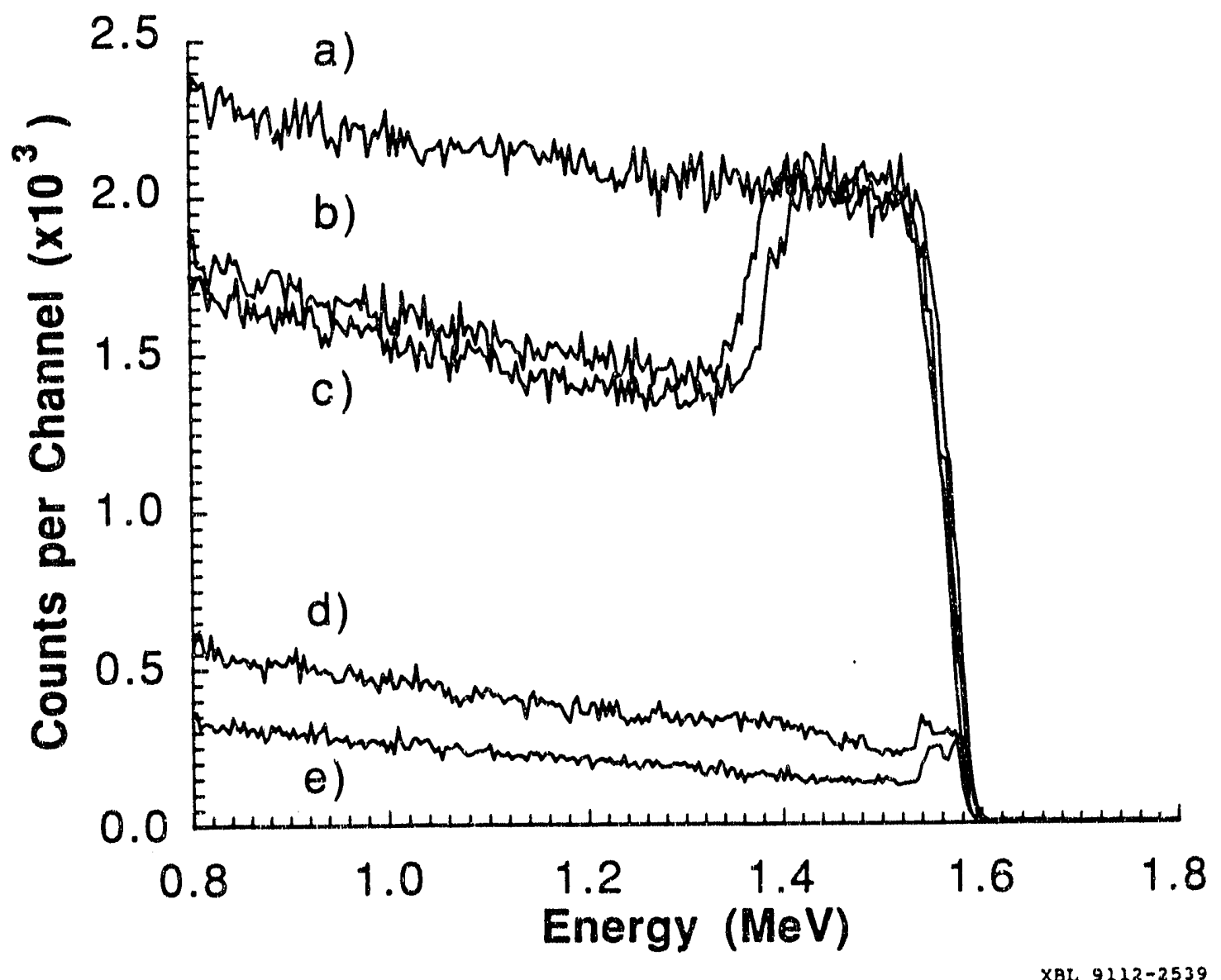

Figure 7.5. $1.95 \mathrm{MeV} \mathrm{He} e^{+}<111>$ aligned RBS spectra for a) random direction, b) C + Ga implant, c) C + Al implant, d) C + B implant, and $\theta$ ) unimplanted sample. The amorphous layer at the surface of the $\mathrm{C}+\mathrm{Ga}$ implanted sample is approximately $140 \mathrm{~nm}$ thick. The amorphous layer at the surface of the C + Al implanted sample is approximately $120 \mathrm{~nm}$ thick. 


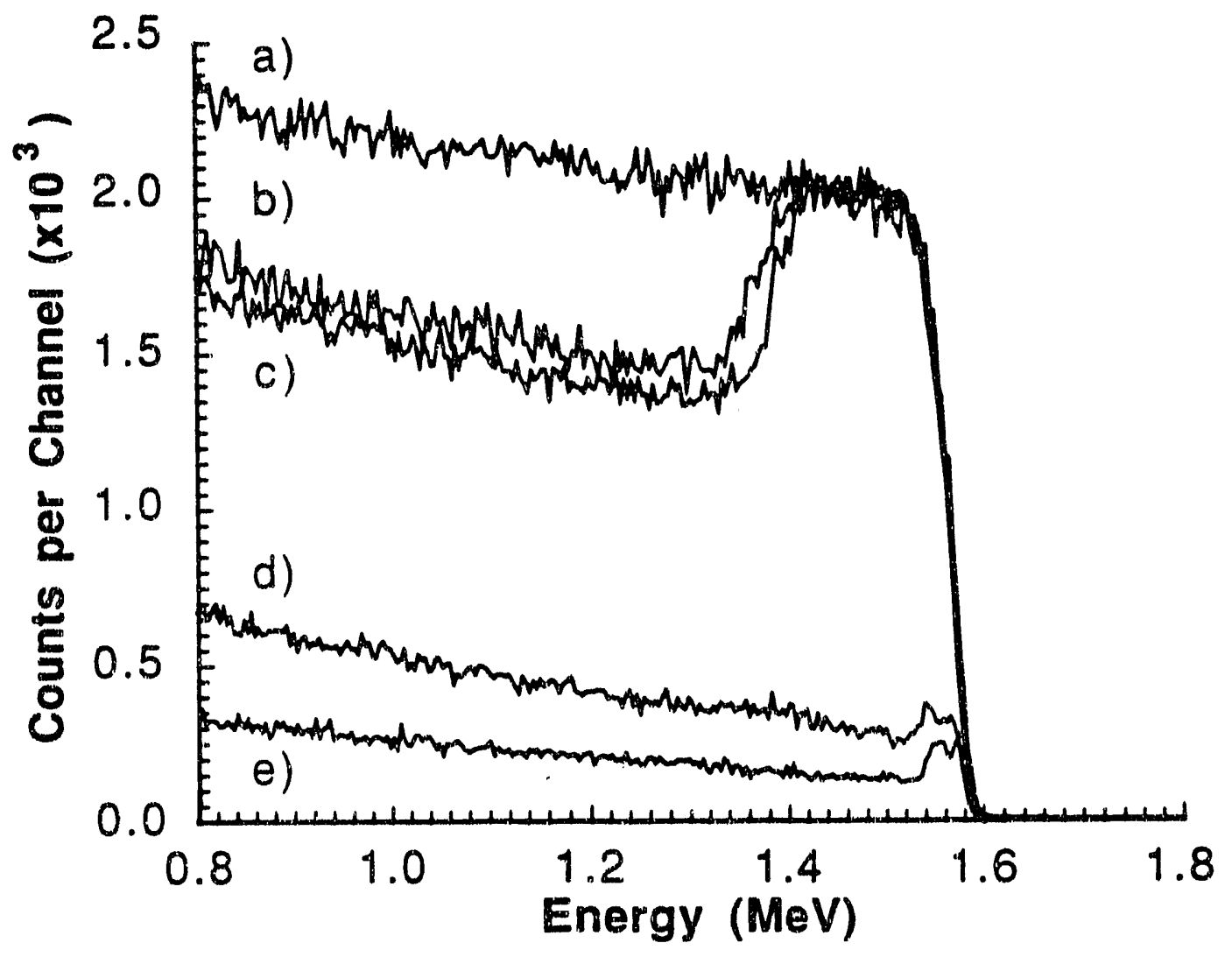

XDL 921-252

Figure 7.6. $1.95 \mathrm{MeV} \mathrm{He}+<111>$ aligned RBS spectra for a) random direction, b) $\mathrm{C}+\mathrm{Kr}$ implant, c) $\mathrm{C}+$ Ar implant, d) $\mathrm{C}$ only implant, and $\theta$ ) unimplanted sample. The amorphous layer at the surface of the $\mathrm{C}+\mathrm{Kr}$ implanted sample is approximately $140 \mathrm{~nm}$ thick. The amorphous layer at the surface of the C + Ar implanted sample is approximately $120 \mathrm{~nm}$ thick. 


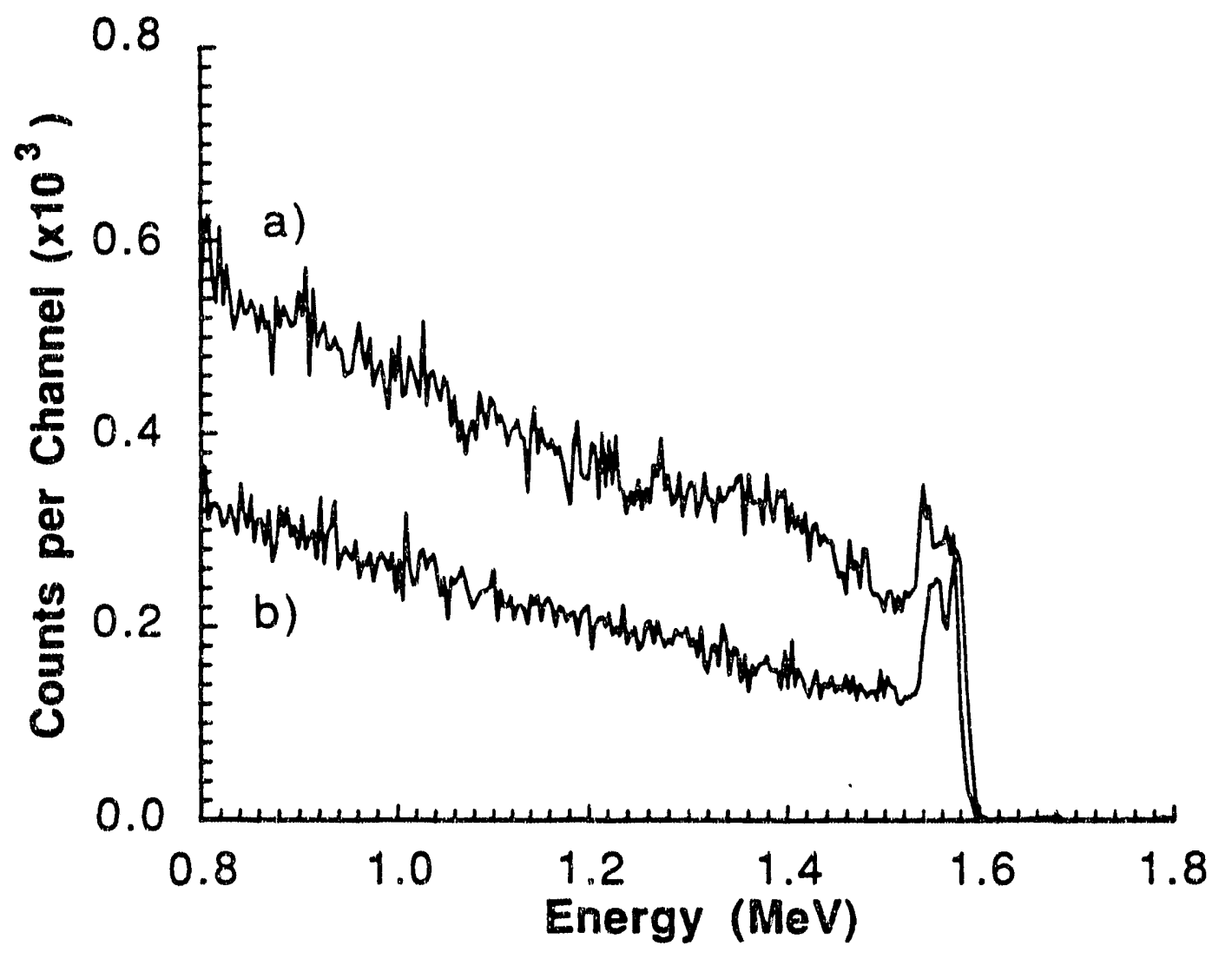

XBL $921-249$

Figure 7.7. $1.95 \mathrm{MeV} \mathrm{He}+<111>$ aligned RBS spectra for a) C implanted alone and b) unimplanted sample. Note the damage at the end of the range of the $\mathrm{C}$ atoms as shown by the increase in backscattered signal. 


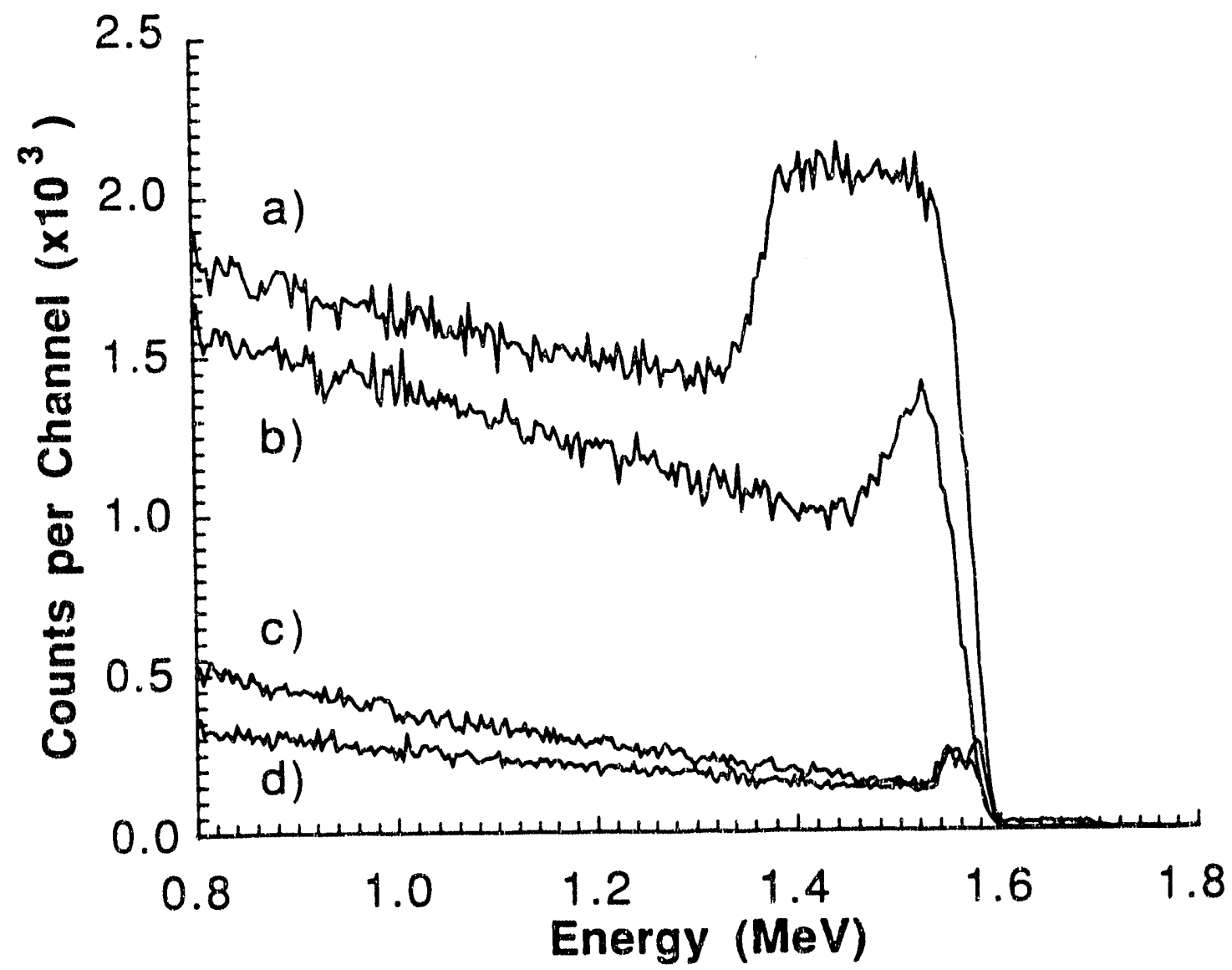

XBL, $9112-2540$

Figure 7.8. $1.95 \mathrm{MeV} \mathrm{He}+<111>$ aligned RBS spectra for layers implanted with $\mathrm{C}$ and $\mathrm{Ga}$ under various annealing conditions: a) as implanted (not annealed), b) annealed at $800^{\circ} \mathrm{C}$ for 10 seconds, c) annealed at $950^{\circ} \mathrm{C}$ for 10 seconds, and d) unimplanted sample. 


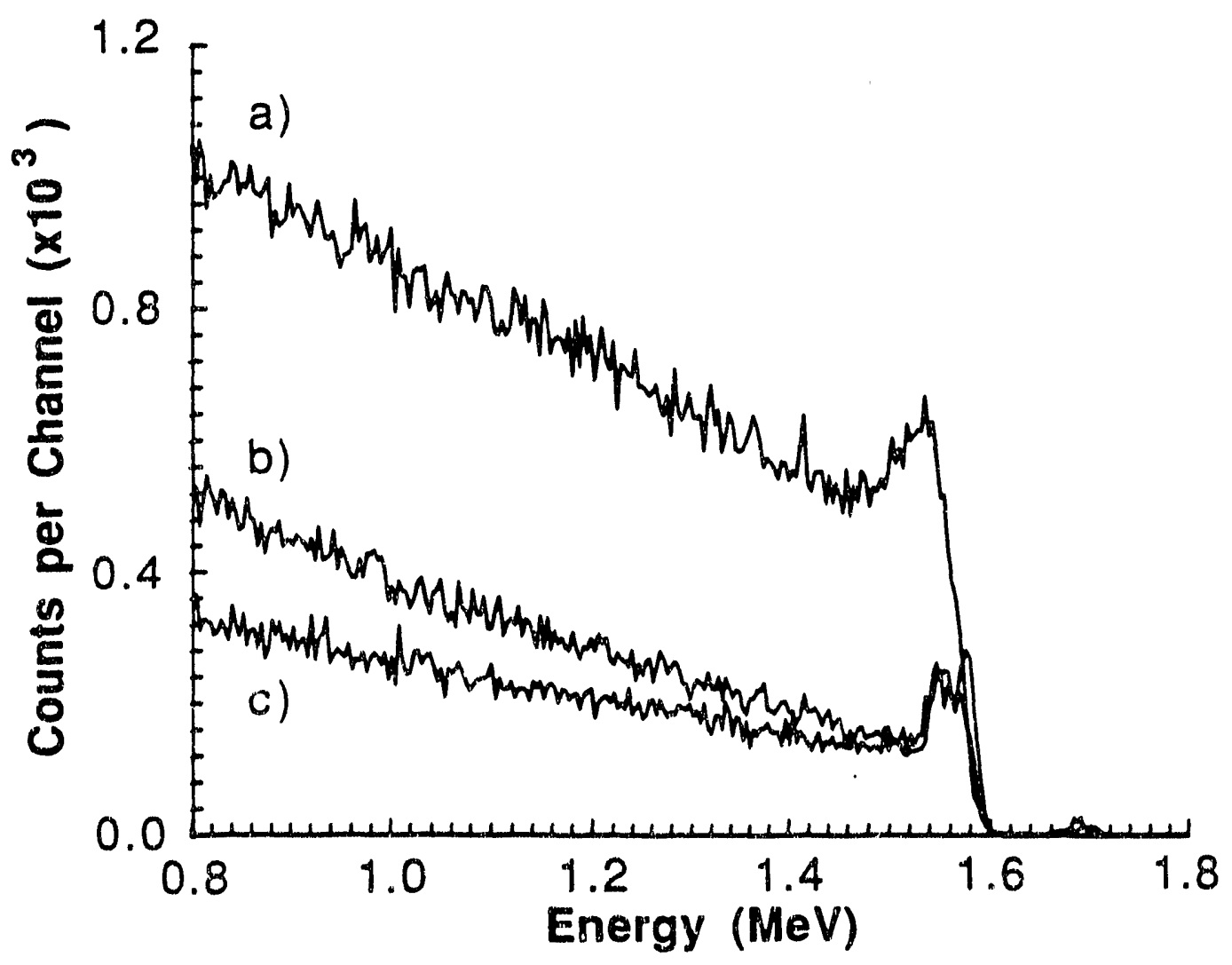

$X B L \quad 921=250$

Figure 7.9. $1.95 \mathrm{MeV} \mathrm{He}+<111>$ aligned RBS spectra for implanted layers following annealing conditions $950^{\circ} \mathrm{C}$ for 10 seconds; a) $\mathrm{C}+\mathrm{Kr}$ implant, b) $\mathrm{C}$ + Ga implant, and c) unimplanted sample. 


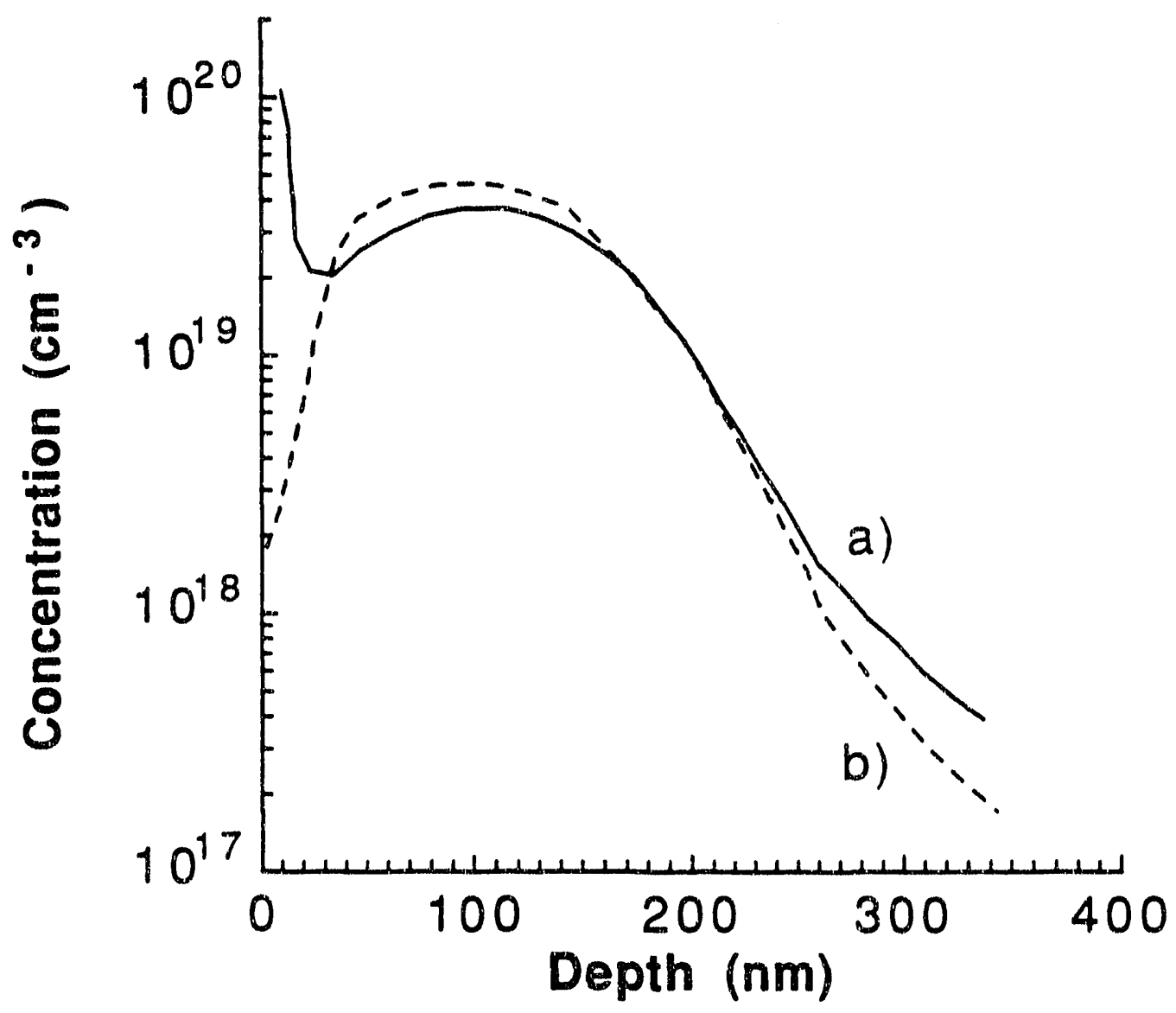

XBL 921-252

Figure 7.10. SIMS spectra of concentration versus depth for samples implanted with $\mathrm{C}+\mathrm{B}$ and annealed at $800^{\circ} \mathrm{C}$ for $10 \mathrm{~s}$. a) [C] and b) [B]. 


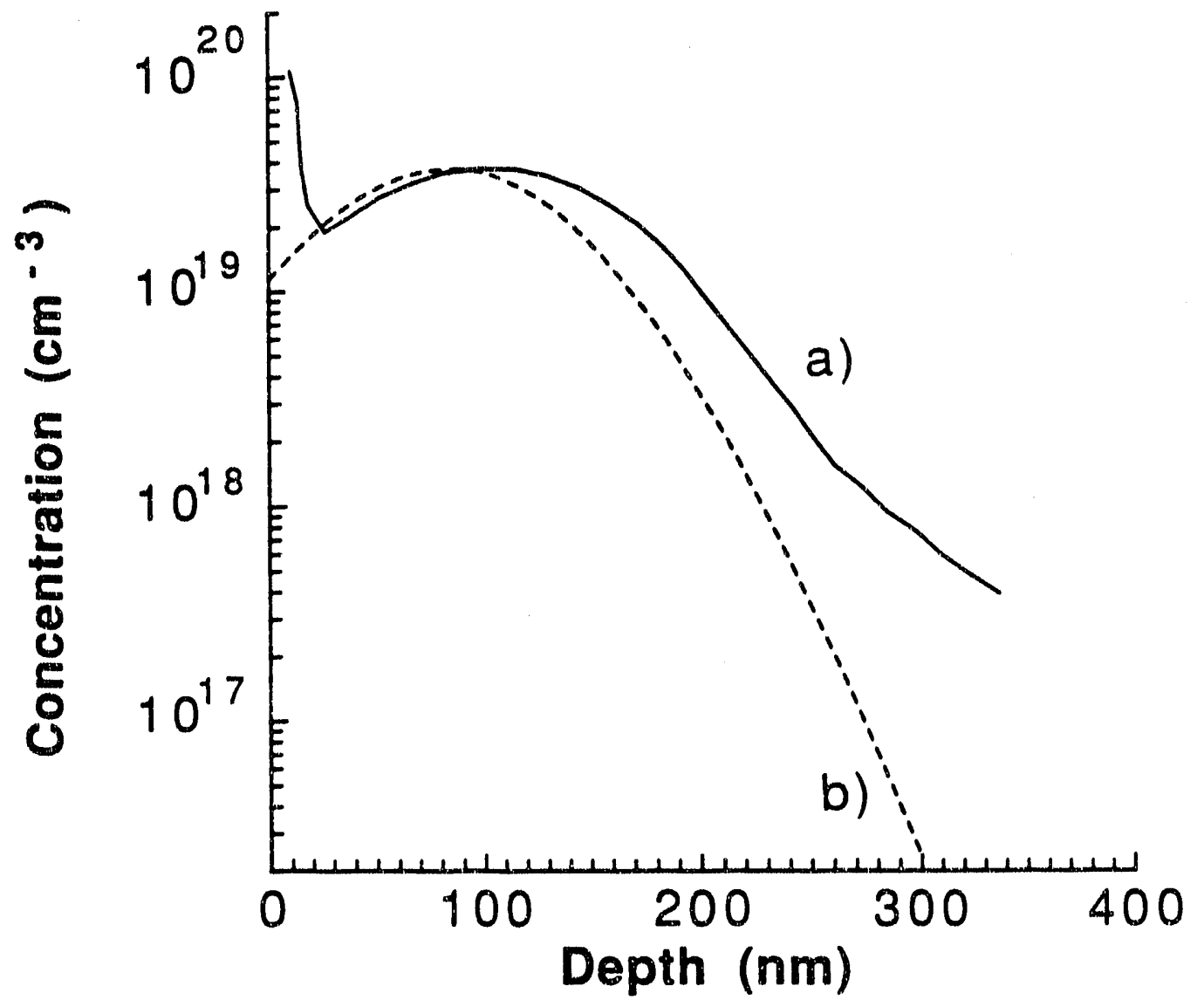

XBL $921-253$

Figure 7.11. Carbon concentration versus depth for a) implanted carbon after annealing at $800^{\circ} \mathrm{C}$ for $10 \mathrm{~s}$. and b)calculated implantation profile. 


\section{DISCUSSION}

Clearly, the increased activation of $\mathrm{C}$ following co-implantation with $\mathrm{Ga}$ is not due to a stoichiometry effect alone. The results presented in this thesis show that increasing the amount of damage in the implanted layer will increase the electrical activatio!?. RBS experiments show that co-implants with ions of atomic weights greater than that of $\mathrm{Al}$ will create an amorphous layer. This damage plays a significant role as is borne out by the increased activation due to both the $\mathrm{Ar}$ and $\mathrm{Kr}$ co-implants. Even As, which should lead to stoichiometry deviations hindering the electrical activation of $\mathrm{C}$, increases the electrical activation to some degree.

However, restoring stoichiometry after $\mathrm{C}$ implantation with a group III coimplant also has a significant effect. The group III elements, compared to other co-implants of similar weight (same row of the periodic table), result in the best electrical activation. Ga co-implants provide the best activation $\left(68 \%\right.$ for $950^{\circ} \mathrm{C}$ anneal, Figure 7.1) of any co-implants used in this study. This result is fully consistent with all other observations. $\mathrm{Ga}$ is native to the GaAs lattice and would be most likely to incorporate onto $\mathrm{Ga}$ sites thereby maintaining stoichiometry during implantation and annealing.

The $\mathrm{C}+\mathrm{B}$ implantation provides a key insight into $\mathrm{C}$ activation. The coimplantation of $B$ appears to have no effect on the implanted layer. The differences in the electrical properties of the $C+B$ implants and the samples implanted with $\mathrm{C}$ alone are statistically insignificant. RBS experiments indicate no additional measurable damage is caused by the co-implant of $B$. These results suggest that the degree of disorder created in the substrate during implantation determines the electrical activity of the $\mathrm{C}$ and that stoichiometry effects alone do not change the activation. B implantation creates no additional damage and therefore does not enhance the electrical activity of the $\mathrm{C}$. 
The substitutional site of the $B$ must also be considered. $B$ easily incorporates onto an As site, 69 and acts as a double acceptor in GaAs. To determine the exact role of the $B$ further experiments need to be conducted examining the electrical characteristics and the substitutional site of the implanted $B$. However, the limited activation of the implanted $C$ shows that $B$ does not aid activation of $\mathrm{C}$ by maintaining stoichiometry and encouraging the C to sit substitutionally on an As site.

It is reasonable to suggest that the ability of the implanted $C$ to sit on an As site and contribute a free hole depends on the degree of disorder in the lattice. C doping during epitaxial growth has been highly successful (as mentioned previously) indicating a natural tendency for $C$ to sit on an As site. The solid phase epitaxy (SPE) during thermal annealing of the amorphous layer created by co-implantation (co-implants with atomic weights greater than that of Al) more closely resembles epitaxial growth of GaAs than the annealing of damage caused by $\mathrm{C}$ implantation. Therefore, we expect $\mathrm{C}_{\mathrm{As}}$ will form more easily in the highly damaged layers.

During SPE stoichiometry is also important. Equal numbers of both constituents at the growth frontier promotes formation of a stoichiometric layer and inhibits the formation of native defects which can compensate dopants. Implantation of a group III atom will result in a "Ga"-rich layer enhancing the incorporation of $\mathrm{C}$ atoms onto As sites. Therefore, heavy group III co-implants result in the best activation. Implanting an equal number of $\mathrm{C}$ and a group III atoms will increase the probability of stoichiometry in the growing layer and reduce the number of native defects. Recent work by Madok and Haegel70 found that co-implants of in also increase the activation of $C$ to nearly $40 \%$.

In comparison, a lightly damaged layer (those implanted with $\mathrm{C}$ only, $\mathrm{C}+$ $B$ and $C+N$ ) still has long range order. The thermodynamics of the lattice will 
constrain the $C$ to sit on an available substitutional site or in a well-defined interstitial position. The chemical nature of the $C$ will not exhibit itself, i.e., the preference of $\mathrm{C}$ to sit on an As site is not the controlling factor in this system.

Alternatively, consider the number of vacancies created during implantation. The light elements, implanted at energies from $30-40 \mathrm{k} \theta \mathrm{V}$ lose energy to electronic processes and the amount of energy lost in nuclear collisions is small. Referring to Figure 2.3 and Table 2.1, we see that for B implanted into GaAs, the energy above which electronic stopping dominates is $13 \mathrm{keV}$. As the implanted atom proceeds through the crystal it loses energy during inelastic scattering. Once its energy is below $13 \mathrm{keV}$ nuclear stopping dominates, however it can only impart a maximum of $13 \mathrm{keV}$ to the atom with which it collides. A majority of the substrate atoms which are displaced during low energy nuclear collisions will come to rest near their original lattice sites. Therefore, during implantation significant self annealing will occur. $71 \mathrm{Ga}$ and As diffuse back to their original lattice sites hence a limited number of vacancies are available for the C. During annealing, the $\mathrm{C}$ must compete with the constituent atoms of the substrate for a substitutional site. Therefore $C$ does not have enough substitutional As sites and could then occupy an available Ga site or in an interstitial position resulting in low activation.

In the case of heavier elements implanted at higher energies ( $>80 \mathrm{keV}$ ) many more $\mathrm{Ga}$ and $\mathrm{As}$ vacancies are created. The energy at which electronic stopping becomes dominate for As implanted into GaAs is $800 \mathrm{keV}$. Ga and As implanted at $180 \mathrm{keV}$ and $220 \mathrm{keV}$ respectively will be principally stopped by nuclear stopping. The higher energy of the collision results in the recoiling atoms have more energy and will travel further from their original lattice positions. Self annealing is not as likely to occur. Enhanced activation of $\mathrm{C}$ 
occurs since more vacancies are available and the $C$ has less competition for a substitutional As site.

Better electrical characteristics are achieved with $950^{\circ} \mathrm{C}$ annealing compared to the $800^{\circ} \mathrm{C}$ annealing in all samples except those implanted with $\mathrm{C}$ $+N$. As carrier concentration increases the mobility is expected to decrease due to ionized impurity scattering. However, hole concentration and mobility are both higher for the $950^{\circ} \mathrm{C}$ anneal than for the $800^{\circ} \mathrm{C}$ anneal. These results indicate that the higher temperature further anneals the implantation dama ie increasing the electrical activation and the mobility. RBS results for samples implanted with $\mathrm{C}$ and $\mathrm{Ga}$ show the extent to which the implantation damage is annealed at the two temperatures (taking into consideration that more than $1 \%$ of the atoms must be displaced to result in a higher dechanneling rate in RBS). The damage has not been completely recovered after $800^{\circ} \mathrm{C}$ annealing but after $950^{\circ} \mathrm{C}$ annealing the substrate is nearly completely recovered. The slightly higher backscattering signal of the implanted and annealed sample compared to a reference (not implanted) sample indicates the existence of extended defects in the implanted substrate. 72

Although a similar amount of damage is caused by the $\mathrm{Kr}$ and $\mathrm{Ga}$ coimplants, more residual defects remain in the $\mathrm{C}+\mathrm{Kr}$ implanted sample following the $950^{\circ} \mathrm{C}$ anneal than in the $\mathrm{C}+\mathrm{Ga}$ implanted sample. I previously stated that the inert gases ( $\mathrm{Ar}$ and $\mathrm{Kr}$ ) will not affect the stoichiometry of the crystal, however they do create disorder in the crystal whether they sit substitutionally or interstitially or form clusters. The effect of these ions is seen in the defects remaining in the substrate following the $950^{\circ} \mathrm{C}$ anneal as shown by the higher backscattered signal. Again, although equal damage is caused by the $\mathrm{Kr}$ and $\mathrm{Ga}$ implantations, the annealing of the lattice is more complete for the $\mathrm{Ga}$ co- 
implant as shown by RBS. This can be expected since $\mathrm{Ga}$ is a constituent of the lattice.

A systematic study to find the optimum annealing conditions has not been conducted. However it is clear that the $800^{\circ} \mathrm{C}, 10$ second anneal is not sufficient for the highly damaged cases. It is likely that extended defects remain which require higher temperatures to dissolve. Pearton et. al.64 attained optimum electrical characteristics following the implantation of $\mathrm{Ga}$ and $\mathrm{C}$ ( $\mathrm{Ga}$ was implanted first) after an $800^{\circ} \mathrm{C}, 10$ second anneal. However, Shin 63 achieved the highest activation following a $900^{\circ} \mathrm{C}$ anneal. The results presented here clearly show a higher temperature anneal is required to fully remove the damage resulting from implantation of heavy ions such as Ga. A more systematic study of the electrical characteristics and damage recovery as a function of annealing parameters is required to determine the optimum annealing conditions for co-implantation of a heavy ion with $\mathrm{C}$.

Why does $\mathrm{C}$ behave so differently than group II acceptors? Consider the difference in activation between C implanted into GaAs and other group II acceptors implanted into GaAs. $\mathrm{Be}$, in particular is a light atom yet will become electrically active without the need for additional damage. Be and the other group $\|$ diffuse very easily by an interstitial mechanism, 73 indicating that the formation of group II interstitials happens readily. Group II interstitials have been identified as donors so their formation is energetically favorable within the native defect model by Walukiewicz. Ga vacancies are acceptors and will not be favorable. Therefore within the implanted layer the reaction of a Be interstitial with a Ga vacancy is energetically favorable and the highly diffusive Be interstitial can quickly diffuse to a site of the reaction. This leads to lower annealing temperatures and higher electrical activation. 
Carbon's low electrical activation can be attributed to several factors. Carbon is highly electronegative and strongly prefers the As site since it does not easily give up an electron although it will readily accept one. Following the same reasoning, one can assume that $C$ interstitials will not act as donors, and therefore Fermi level stabilization will not drive the formation of $C$ interstitials. As vacancies are donors so their presence is energetically favorable (again considering the native defect model). The small diffusion coefficient of $\mathrm{C}$ indicates that if an As vacancy is not in the vicinity of the final resting location of the $C$ atom, it is unlikely the $C$ will diffuse to an As vacancy. All of these arguments suggest that $C$ activation is unfavorable relative to the activation of implanted group $1 /$ elements.

It is also interesting to compare the behavior of implanted $\mathrm{C}$ with that of $\mathrm{Si}$, a group IV donor, which occupies the $\mathrm{Ga}$ site. Si, is of course heavier and will cause more damage, resulting in more vacancies and interstitials. Si also has been shown to occupy either the Ga site or the As site particularly at high doping levels leading to self compensation. Si has not been shown to be a particularly fast diffuser therefore it would appear the higher activation is due primarily to the increased amount of damage.

The question remains as to where the inactive $C$ is. Is it interstitial, segregated to extended defects (unlikely due to small amount of damage caused by the $C$ only implant and low diffusivity of $C$ ), self compensating due to $\mathrm{C}_{\mathrm{Ga}}$, neutral interstitials, compensated by other native defects, or perhaps in clusters (unlikely due to low diffusivity of $\mathrm{C}$ )? One method of examining this issue is to observe the local vibrational mode spectra of implanted samples with varying activation efficiencies. Concentration of $\mathrm{C}_{\mathrm{As}}$ can be determined by integrating under the peak due to the local vibrational mode. Comparing the $C_{A s}$ local vibrational mode of a two samples with the same $C$ concentration but 
different electical activity could provide a clue to the location of the inactive $\mathrm{C}$. One difficulty in this experiment is background $C$ in the substrate which in semiinsulating GaAs is usually present in a concentration of about $10^{15} \mathrm{~cm}^{-3}$. Clearly, further work is required in this field. 


\section{CONCLUSIONS}

Implantation in GaAs leads to a series of intricate processes. The interaction of the implanted species, native defects, and deviations from stoichiometry are interactive and complicated. However this study shows that co-implantation plays a dual role in the increased electrical activation of implanted C. The co-implant must cause considerable damage to the lattice and maintain stoichiometry to optimize the electrical activation of $\mathrm{C}$. Investigations are continuing to determine the optimum annealing temperature for the activation of $\mathrm{C}$ and the effect of changing the range of the co-implant on the electrical characteristics of implanted $\mathrm{C}$. 


\section{REFERENCES}

4. E. Rutherford, Phil. Mag. 21, 212 (1911).

2. N. Bohr, Phil Mag. 30, 581 (1915).

3. G. Carter and W. A. Grant, lon Implantation in Semiconductors (Wiley and Sons, New York, 1976).

4. H. Ryssel and I. Ruge, Ion Implantation (Wiley and Sons, New York, 1986).

5. J. Lindhard, M. Scharf, H.E. Schiott, Kgl. Danske. Videnskab. Selskab. Mat.Fys. Medd. 33 (1963) No. 14.

6. G.H. Kinchin and R.S. Pease, Rep. Progr. Phys. 18, 1 (1955).

7. J.S. Harris in Ion Implantation in Semiconductors, eds. I. Ruge and J. Graul (Springer-Verlag, New York, 1971) p. 157.

8. J. E. Westmoreland, O.J. Marsh, R.G. Husperger, Radiat. Eff. 5, 2.45 (1970).

9. J. P. Donnelly, Nucl. Inst. Meth. 182, 553 (1981).

10. R.T. Blunt in Solid State Devices 1985, eds. P. Balk and O.G. Folberth (Elselvier Science, The Netherlands, 1986) p. 133.

11. J.P. Donnelly, W.T. Lindley, and C.E. Hurwitz, Appl. Phys. Lett. 27, 41 (1975).

12. H. Kanber, R.J. Cipolli, W.B. Henderson, and J.M. Whelan, J. Appl. Phys. $57,4732(1985)$.

13. L. Palmetshofer, J. Kastener, and K. Lubke, Nucl. Instr. and Meth. B59/60, 1081 (1991).

14. M. Kuzuhara, T. Nazaki, and H. Kohzu, J. Appl. Phys. 58, 1204 (1985).

15. K. K. Patel, R. Bensalem, M.A. Shahid and B.J. Sealy, Nucl. Instr. Meth. $B 7 / 8,418(1985)$.

16. S.S. Gill, Solid State Phenomena, 1\&2, 281 (1988).

17. D.E. Davies and P.J. McNally, Appl. Phys. Lett. 44, 304 (1984).

18. F. Sette, S.J. Pearton, J.M. Poate, and J.E. Rowe, Nucl. Instrum. Methods B19/20, 408 (1987).

19. V.U. Galavanov, S.G. Metreveli, N.V. Siukaev and S.P. Staroseltsova, Sov. Phys. Sernicon. 3, 94 (1969). 
20. W. Walukiewicz, Appl. Phys. Lett. 54, 2094 (1989).

21. W. Walukiewicz, J. Vac. Sci. Technol. B5, 1062 (1987).

22. W. Walukiewicz, Phys. Rev. B37, 4760 (1988).

23. R. Heckingbottom and T. Ambridge, Radiat. Eff. 17, 31 (1973).

24. F. Hyuga, H. Yamazaki, K. Watanabe, and J. Osaka, Appl. Phys. Lett. 50, $1592(1987)$.

25. A.R. Von Neida, S.J. Pearton, M. Stavola, R. Caruso, Appl. Phys. Lett. 49, 1708 (1986).

26. E.B. Stoneham, G.A. Patterson, and J.M. Gladsturie, Radiat. Eff. 47, 143 (1980).

27. S. Sugitani, F. Hyuga, and K. Yamasaki, J. Appl. Phys. 67, 552 (1990).

28. W.D. Fan and W.Y. Wang, Nucl. Instr. and Meth. 859/60, 1086 (1991).

29. T. Inada, S. Kato, T. Ohkubo, and T. Hara, Radiat. Eff. 48, 91 (1980).

30. C. A. Stolte in Ion Implantation in Semiconductors ed. by F. Chernow, J.A. Borders and D.K. Brice (Plenum Press, NY), p. 149.

31. A.C.T. Tang, B.J. Sealy, and A.A. Rezazadeh, Vacuum 39, 1061 (1989).

32. K.K. Patel, and B.J. Sealy, Appl. Phys. Lett. 48, 1467 (1986).

33. J. Kasahara, K. Taira, Y. Kato, M. Arai, and N. Watanabe, Jpn. J. of Appl. Phys. 22, L373 (1983).

34. A.R. Von Neida, S.J. Pearton, M. Stavola, and R. Caruso, Appl. Phys. Lett. 49, 1708 (1986).

35. L.A. Christel and J.F. Gibbons, J. Appl. Phys. 52, 5050 (1981).

36. T.E. Haynes and O. W. Holland, Appl. Phys. Lett. 59, 452 (1991).

37. T.E. Haynes and O.W. Holland, Nucl. Instr. Meth. B59/60, 1028 (1991).

38. F.G. Moore and H.B. Dietrich, Nucl. Instr. Meth. B59/60, 978 (1991).

39. B.L. Crowder, J. Electrochem. Soc. 117, 671 (1970).

40. W.G. Opyd, J.F. Gibbons, J.C. Bravman, and M.A. Parker, Appl. Phys. Lett. $49,974(1986)$. 
41. Y.I. Nissim, L.A. Christel, T.W. Sigmon, J.F. Gibbons, T.J. Magee, and R. Ormond, Appl. Phys. Lett. 39, 598 (1981).

42. W.G. Opyd, J.F. Gibbons, and A.J. Mardinly, Appl. Phys. Lett. 53, 1515 (1988).

43. W.G. Opyd and J.F. Gibbons, J. Appl. Phys. 67, 7417 (1990).

44. S.J. Pearton, J.M. Poate, F. Sette, J.M. Gibson, D.C. Jacobson, and J.S. Williams, Nucl. Inst. Meth. B19/20, 369 (1987).

45. T. Haga, N. Tachino, Y. Abe, J. Kasahara, A. Okubor, and H. Hasegawa, J. Appl. Phys. 66, 5809 (1989).

46. A.M. White, P.J. Dean, D.J. Ashen, J.B. Mullin, M. Webb, B. Day, and P.D. Greene, J. Physics C 6, L243 (1973).

47. R.C. Newman, F. Thompson, M. Hyliands, and R.F. Peart, Solid State Comm. 10, 505 (1972).

48. K. Woodhouse, R.C. Newman, T.J. de Lyon, J.M. Woodall, G.J. Scilla, and F. Cardone, Semicond. Sci. Tech. 6, 330 (1991).

49. P.M. Enquist, J.A. Hutchby, and T.J. de Lyon, J. Appl. Phys. 63, 4485 (1988).

50. M. llegems, J. Appl. Phys. 48, 1278 (1977).

51. M.B. Das, IEEE Trans. Electron Devices 35, 604 (1988).

52. B.T. Cunningham, L.J. Guido, J.E. Baker, J.S. Major, Jr., N. Holonyak, Jr., and G.E. Stillman, Appl. Phys. Lett. 55, 687 (1989).

53. C.R. Abernathy, S.J. Pearton, F. Ren, W.S. Hobson, T.R. Fullowan, A. Katz, A.S. Jordan and J. Kovalchik, J. of Cryst. Growth 105, 375 (1990).

54. T.J. de Lyon, J.M. Woodall, M.S. Goorsky, and P.D. Kirchner, Appl. Phys. Lett. 56, 1040 (1990).

55. T. Yamada, E. Tokumitsu, K. Saito, T. Akatsuka, M. Komagai, and K. Takahashi, J. Cryst. Growth 95, 145 (1989).

56. T.J. de Lyon, J.M. Woodall, M.S. Goorsky, and P.D. Kirchner, Appl. Phys. Lett. 56, 1040 (1990).

57. M.C. Hanna, Z.H. LU, and A. Majerteld, Appl. Phys. Lett. 58, 164 (1991).

58. M.C. Hanna, Z.H. Lu, E.W. Mao, T. McCormick, E.G. Oh, A. Majerfeld, J. of Cryst. Growth 107, 279 (1991). 
59. J.D. Sansbury and J.F. Gibbons, Radiat. Eff. 6, 269 (1970).

60. J.S. Harris in International Conference on lon Implantation in Semiconductors, edited by I. Ruge and J. Graul (Springer-Verlag, Berlin, 1971) p. 157.

61. B.K. Shin, Appl. Phys. Lett. 29, 438 (1976)

62. W.M. Paulson and G. Tam in Semi-Insulation III-V Materials 1984, edited by D.C. Look and J.S. Blakemore (Shiva, Cheshire, England, 1984) p. 53.

63. B.K. Shin, J.E. Ehret, Y.S. Park, and M. Stefiniw, J. Appl. Phys. 49, 2988 (1978).

64. S.J. Pearton and C.R. Abernathy, Appl. Phys. Lett. 55, 678 (1989).

65. T. Sands, D.K. Sadana, R. Gronsky, and J. Washburn, Appl. Phys. Lett. 44, 874 (1984).

66. S.J. Pearton, W.S. Hobson, A.P. Kinsella, J. Kovalchick, U.K. Chakrabarti, and C.R. Abernathy, Appl. Phys. Lett. 56, 1263 (1990).

67. B. Molnar, Appl. Phys. Lett. 36, 927 (1980).

68. L.J. Van der Pauw, Philips Res. Report 13, 1 (1958).

69. J. Woodhead, R.C. Newman, I. Grant, D. Rumsby, and R.M. Ware, J. Phys. C 16, 5523, (1983).

70. J.H. Madok and N.M. Haegel, Proc. Mat. Sci. Vol. 240, 777 (1992).

71. G. Carter, I.S. Tashlykov, and M.J. Nobes, Rad. Eff. Lett. 85, 37 (1984).

72. M.G. Grimaldi, B.M. Paine, M-A. Nicolet, and D.K. Sadana, J. Appl. Phys. 52, 4038 (1981).

73. U.M. Gösele, Ann. Rev. Mater. Sci., 18, 257 (1988).

74. S.J. Pearton, Sol. St. Phen. 1\&2, 247 (88). 
Appendix I: Summary of implantation schedules and results from the literature.

\begin{tabular}{|c|c|c|c|c|c|}
\hline $\begin{array}{l}\text { Energy } \\
(k \theta V)\end{array}$ & $\begin{array}{l}\text { Dose } \\
\left(\mathrm{cm}^{-2}\right)\end{array}$ & Ion & $\begin{array}{l}\text { Anneal } \\
\text { Temp. } \\
\left({ }^{\circ} \mathrm{C}\right)\end{array}$ & $\begin{array}{l}\text { Sheet Carr. } \\
\text { Conc. } \\
\left(\mathrm{cm}^{-2}\right)\end{array}$ & $\begin{array}{l}\text { Activation } \\
\text { Efficiency } \\
(\%)\end{array}$ \\
\hline 70 & $1 \times 10^{13}$ & $C$ & 700 & $2 \times 10^{11}$ & 2 \\
\hline 70 & $1 \times 10^{14}$ & $C$ & 700 & $1 \times 10^{11}$ & 1 \\
\hline 70 & $1 \times 10^{15}$ & $C$ & 700 & $6 \times 10^{11}$ & .1 \\
\hline 200 & $2 \times 10^{14}$ & $C$ & 800 & $3 \times 10^{12}$ & 1.5 \\
\hline 2.00 & $2 \times 10^{14}$ & $c$ & 800 & $1.4 \times 10^{13}$ & $7^{\star \prime}$ \\
\hline 120 & $1 \times 10^{13}$ & $\mathrm{C}$ & 900 & $5 \times 10^{12}$ & 50 \\
\hline 120 & $1 \times 10^{14}$ & $C$ & 900 & $1.2 \times 10^{13}$ & 12 \\
\hline 60 & $1 \times 10^{14}$ & $C$ & 700 & $3.6 \times 10^{12}$ & 3.6 \\
\hline $\begin{array}{l}60 \\
120\end{array}$ & $\begin{array}{l}1 \times 10^{14} \\
1 \times 10^{14}\end{array}$ & $\begin{array}{l}\mathrm{C} \\
\mathrm{Ga}\end{array}$ & 700 & $1.2 \times 10^{13}$ & 12 \\
\hline 60 & $1 \times 10^{14}$ & $C$ & 900 & $9.5 \times 10^{12}$ & 9.5 \\
\hline $\begin{array}{l}60 \\
120\end{array}$ & $\begin{array}{l}1 \times 10^{14} \\
1 \times 10^{14}\end{array}$ & $\begin{array}{l}\mathrm{C} \\
\mathrm{Ga}\end{array}$ & 900 & $3.2 \times 10^{13}$ & 32 \\
\hline 80 & $3 \times 10^{13}$ & $C$ & 850 & $2.7 \times 10^{12}$ & 9 \\
\hline 80 & $5 \times 10^{13}$ & $c$ & 850 & $2.7 \times 10^{12}$ & 5 \\
\hline $\begin{array}{l}50 \\
150\end{array}$ & $\begin{array}{l}5.7 \times 10^{11} \\
16.4 \times 10^{11}\end{array}$ & $\begin{array}{l}\mathrm{C} \\
\mathrm{C}\end{array}$ & 850 & $6.0 \times 10^{11}$ & 27 \\
\hline
\end{tabular}

"Sample implanted at $77 \mathrm{~K}$ 


\begin{tabular}{|c|c|c|c|c|c|}
\hline $\begin{array}{l}\text { Energy } \\
(\mathrm{k} \in \mathrm{V})\end{array}$ & $\begin{array}{l}\text { Dose } \\
\left(\mathrm{cm}^{-2}\right)\end{array}$ & Ion & $\begin{array}{l}\text { Anneal } \\
\text { Temp. } \\
\text { (OC) }\end{array}$ & $\begin{array}{l}\text { Sheet Carr. } \\
\text { Conc. } \\
\left(\mathrm{cm}^{-2}\right)\end{array}$ & $\begin{array}{l}\text { Activation } \\
\text { Efficiency } \\
(\%)\end{array}$ \\
\hline 40 & $1 \times 10^{13}$ & C & 800 & $3.4 \times 10^{12}$ & 34 \\
\hline $\begin{array}{l}40 \\
180\end{array}$ & $\begin{array}{l}1 \times 10^{13} \\
1 \times 10^{13}\end{array}$ & $\begin{array}{l}\mathrm{C} \\
\mathrm{Ga}\end{array}$ & 800 & $6 \times 10^{12}$ & 60 \\
\hline 40 & $5 \times 10^{14}$ & C & 800 & $1.3 \times 10^{12}$ & 2.5 \\
\hline $\begin{array}{l}40 \\
180\end{array}$ & $\begin{array}{l}5 \times 10^{14} \\
5 \times 10^{14}\end{array}$ & $\begin{array}{l}\mathrm{C} \\
\mathrm{Ga}\end{array}$ & 800 & $2.1 \times 10^{14}$ & 43 \\
\hline
\end{tabular}

\section{References}

[1] J.D. Sansbury and J.F. Gibbons, Radiat. Eff. 6, 269 (1970).

[2] J.S. Harris, in International Conference on Ion Implantation in

Semiconductors, edited by I. Ruge and J. Graul (Springer-Verlag, Berlin, 1971),

p.157.

[3] B.K. Shin, Appl. Phys. Lett., 29, 438 (1976).

[4] B.K. Shin, J.E. Ehret, Y.S. Park, and M. Stefiniw, J. Appl. Phys. 49, 2988 (1978).

[5] W.M. Paulson and G. Tam, in Semi-Insulating III-V Materials 1984, edited by D.C. L.ook and J.S. Blakemore (Shiva, Cheshire, England 1984) p. 53.

[6] S.J. Pearton and C.R. Abernathy, Appl. Phys. Lett. 55, 678 (1989). 
Appendix II: Implantation Profiles

Ion profiles calculated using LSS theory for implantation parameters used in this thesis.
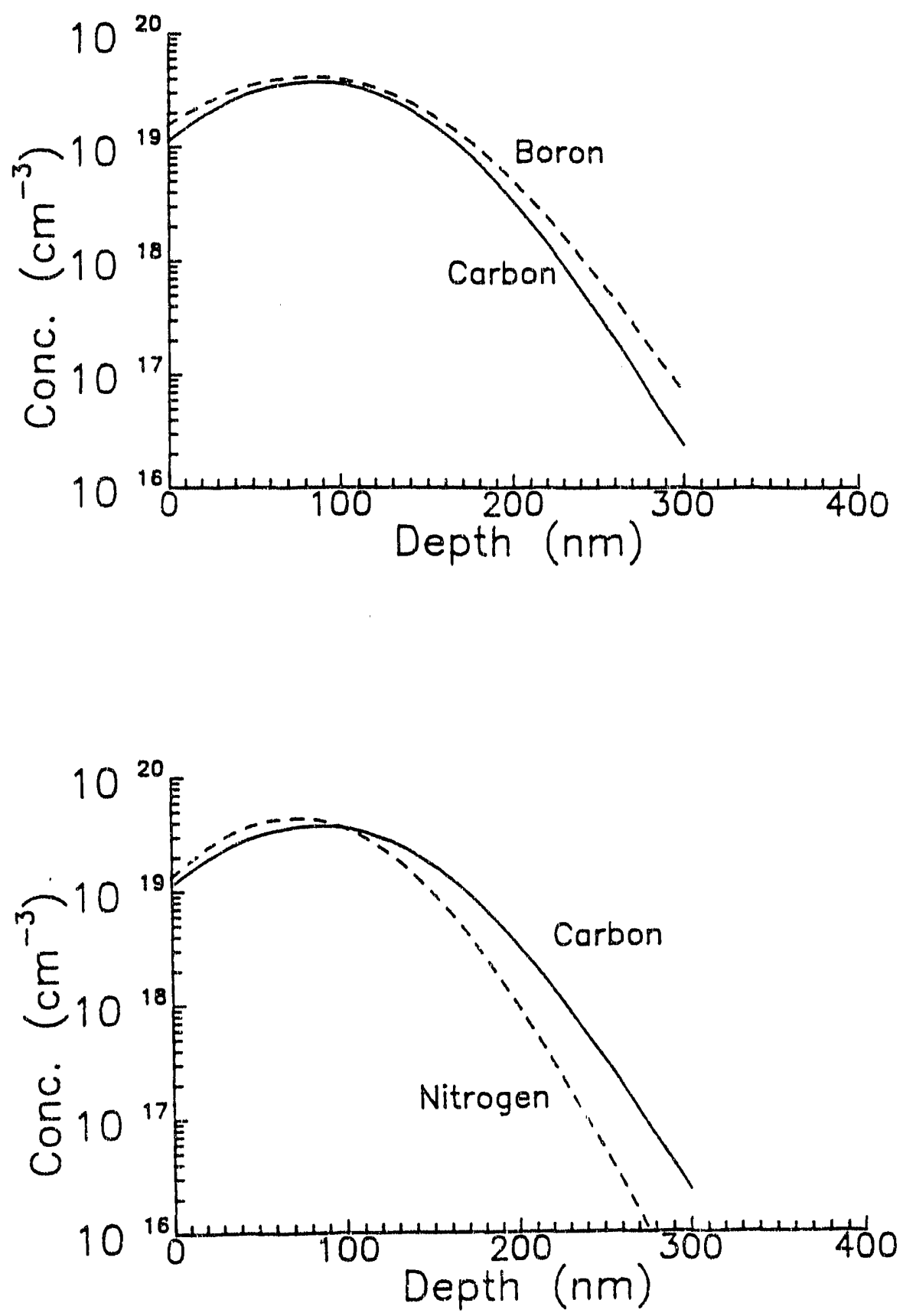

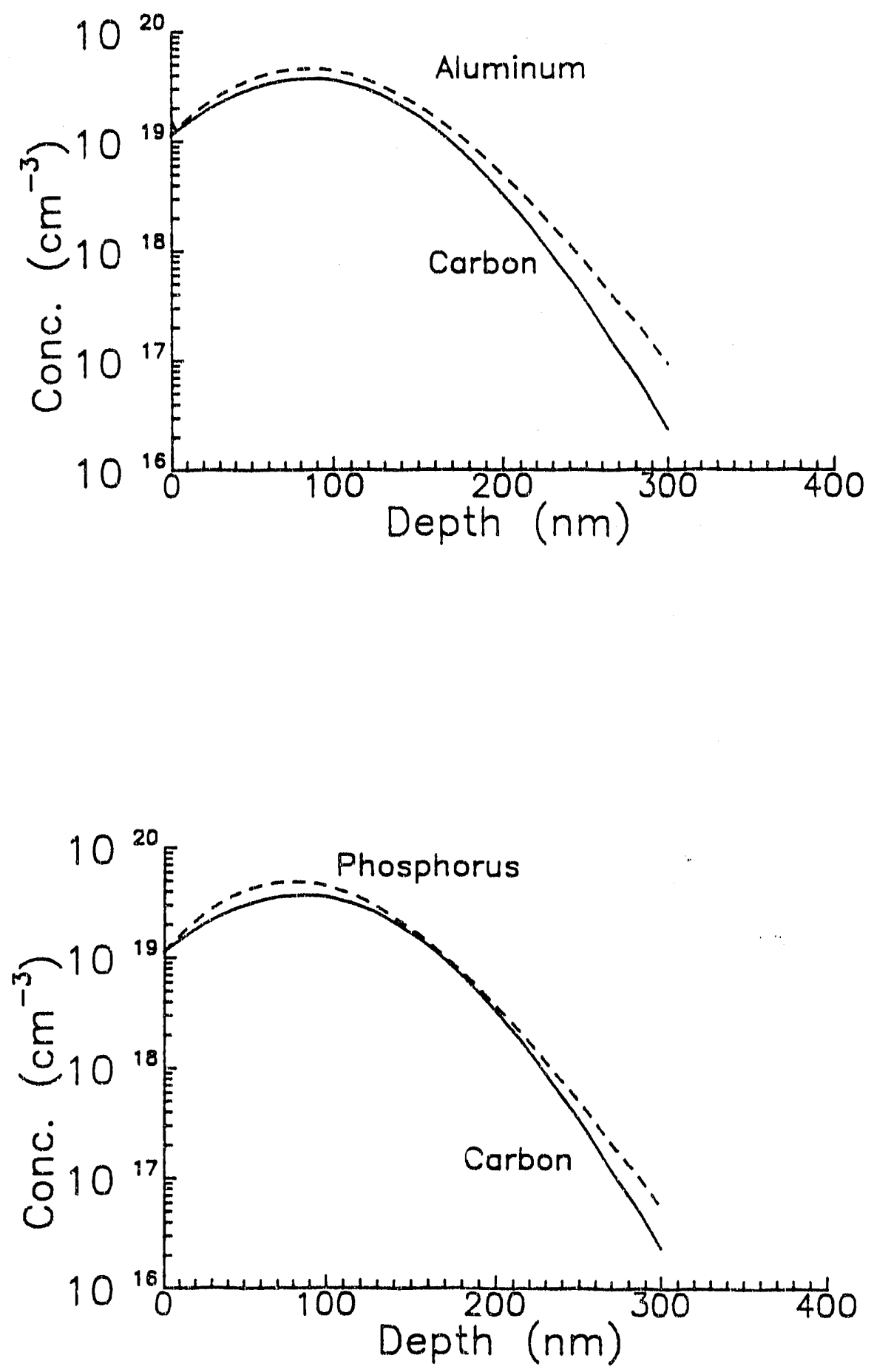

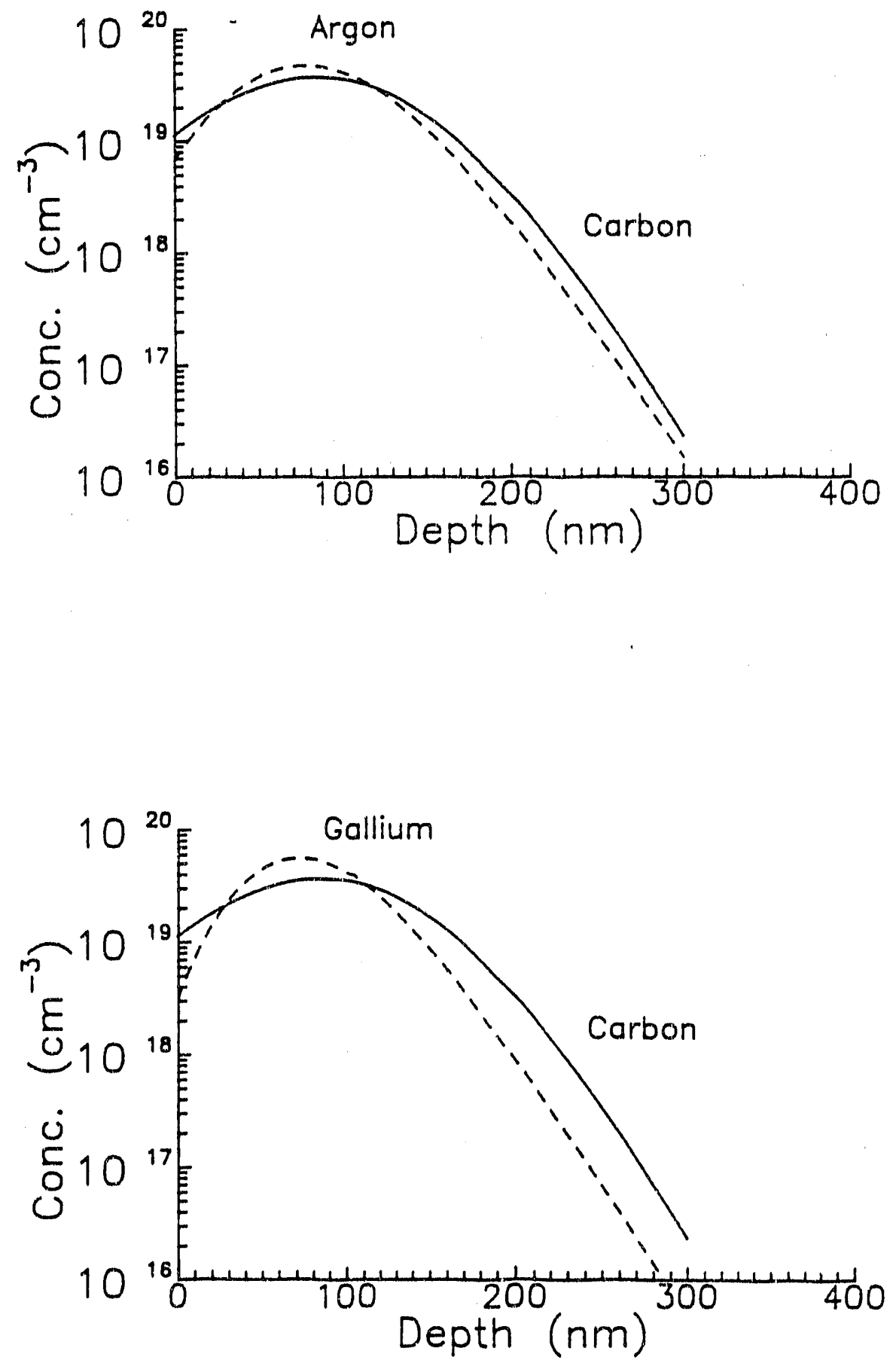

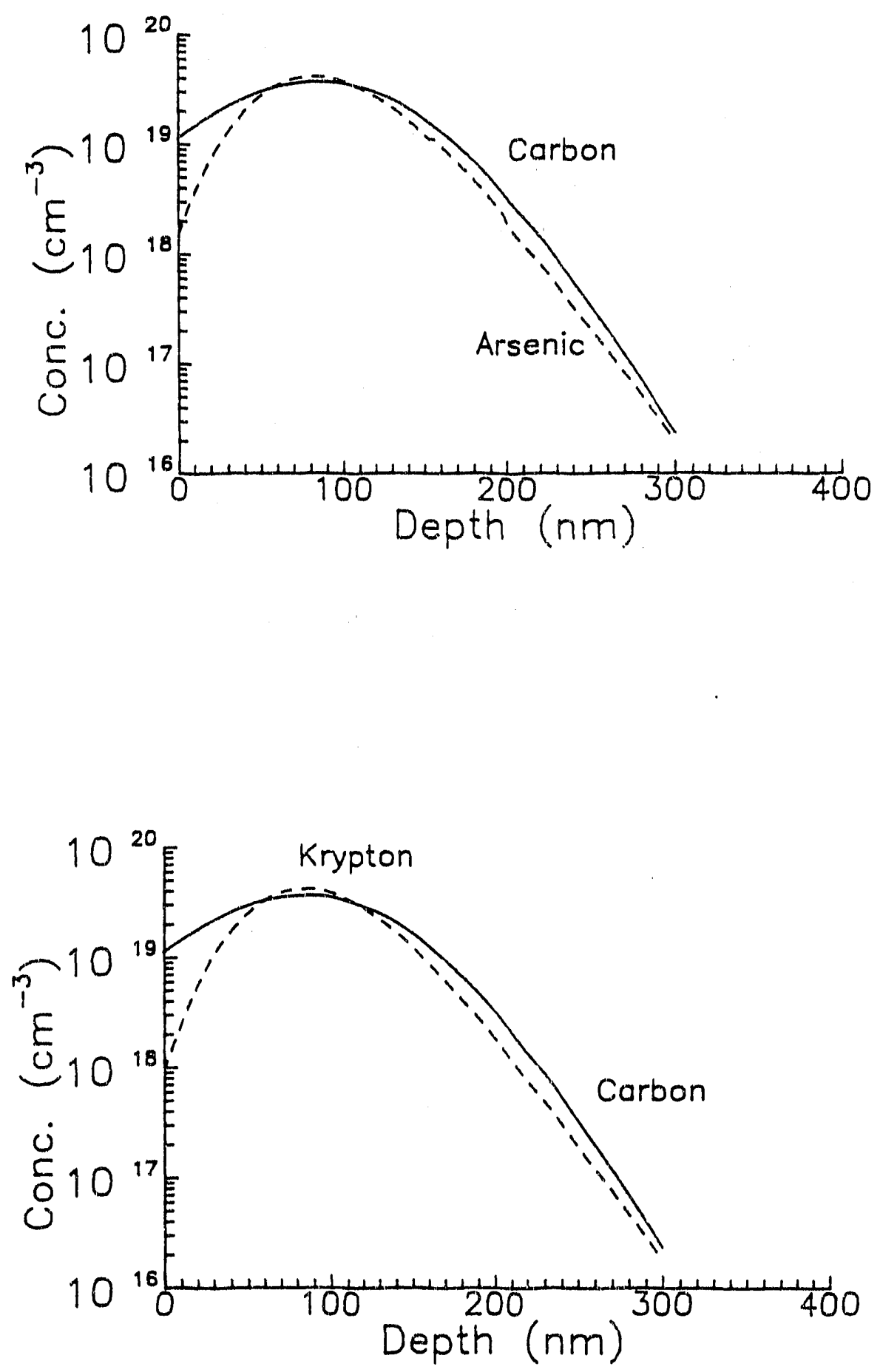
Appendix III: Complete Hall effect data for all samples.

Table A: Samples annealed at $8000^{\circ} \mathrm{C}$

Sample Co-implant Sheet Carr. Conc. $\left(\mathrm{cm}^{-2}\right)$

$4.0 \times 10^{12} \quad 0.8$

$6.4 \times 1012 \quad 1.3$

$4.7 \times 10^{12}$

0.9

$7.3 \times 10^{12}$

1.5

$7.0 \times 10^{12} \quad 1.4$

$2.1 \times 10^{14} \quad 42.8$

$2.2 \times 10^{14} \quad 43.2$

$1.5 \times 10^{14}$

29.8

$3.4 \times 10^{12}$

$3.9 \times 1012$

$1.6 \times 1012$

$8.5 \times 10^{12}$

$4.6 \times 10^{13}$

$5.0 \times 1012$

$3.6 \times 10^{12}$

$1.1 \times 10^{12}$

0.2

$4.1 \times 10^{11}$

0.1

0.7

$\mathrm{Kr} 1-7-29 \quad \mathrm{Kr}$

Ar1-7-26 Ar

Ar2-7-26 Ar
Sheet

Resist.

$\left(10^{3} \Omega / \mathrm{a}\right) \quad\left(\mathrm{cm}^{2} \mathrm{~N} \mathrm{~s}\right)$

Mob.

Efficiency

17.2

90

6.8

144

8.3

161

6.4

133

5.4

164

0.51

57

0.63

46

0.58

72

10.3

178

11.0

147

30.4

127

7.3

101

1.9

73

13.9

93

13.7

128

47.9

122

99.8

153

$1.2 \times 10^{12} \quad 24.0$

$4.4 \times 10^{13}$

8.8

$8.5 \times 10^{13} \quad 17.0$

1.0

50

2.65

101

1.30

54 
Table B: Samples annealed at $950^{\circ} \mathrm{C}$.

$\begin{array}{llllll}\text { Sample } & \text { Co-implant } & \begin{array}{l}\text { Sheet Carr. } \\ \text { Conc. } \\ \left(\mathrm{cm}^{-2}\right)\end{array} & \begin{array}{l}\text { Activ. } \\ \text { Efficiency } \\ (\%)\end{array} & \begin{array}{l}\text { Sheet } \\ \text { Resist. } \\ \left(10^{3} \Omega / \mathrm{a}\right)\end{array} & \text { Mob. } \\ & & \left(\mathrm{cm}^{2} \mathrm{~N} \mathrm{~s}\right) & \\ \text { 2-7-31 } & \text { none } & 1.7 \times 10^{13} & 3.4 & 2.39 & 152 \\ \text { Ga6-3-29 } & \mathrm{Ga} & 3.4 \times 10^{14} & 68.0 & 0.25 & 46 \\ \text { Al3-8-27 } & \mathrm{Al} & 1.7 \times 10^{14} & 34.2 & 0.58 & 72 \\ \text { B3-7-26 } & \mathrm{B} & 2.4 \times 10^{13} & 4.8 & 1.51 & 174 \\ \text { As2-9-17 } & \text { As } & 7.9 \times 10^{13} & 15.8 & 0.76 & 104 \\ \text { P3-7-26 } & \mathrm{P} & 5.4 \times 10^{12} & 1.1 & 8.88 & 129 \\ \text { N3-7-26 } & \mathrm{N} & 7.5 \times 10^{11} & 0.2 & 68.5 & 121 \\ \text { Kr2-7-29 } & \mathrm{Kr} & 1.6 \times 10^{14} & 32.0 & 0.55 & 72 \\ \text { Ar2-9-12 } & \text { Ar } & 6.2 \times 10^{13} & 12.4 & 1.05 & 96 \\ \text { Ar4-9-12 } & \text { Ar } & 6.1 \times 10^{13} & 12.2 & 0.97 & 106\end{array}$




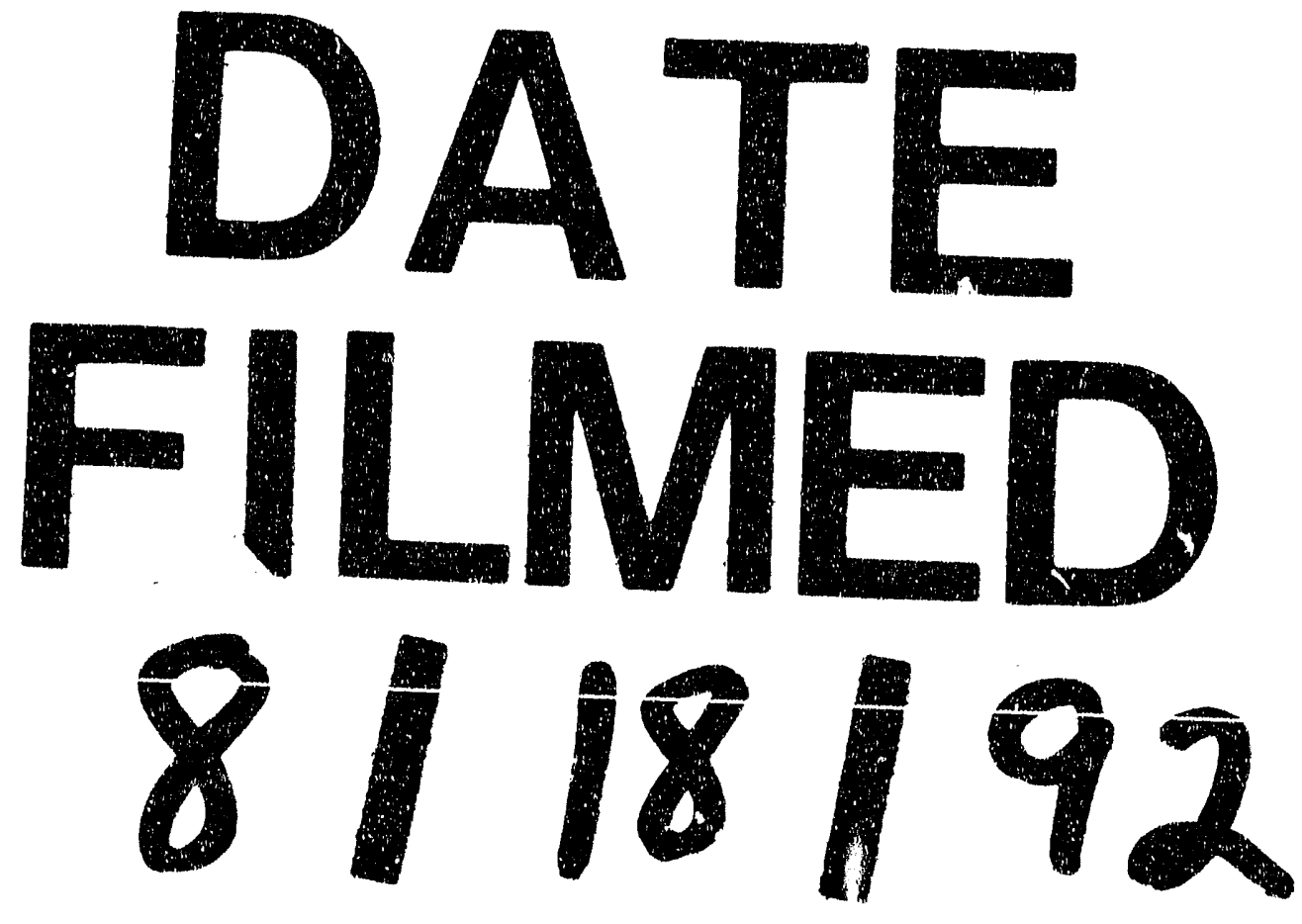


$=\overline{1}$
$=$
$=$
$=$
$=$ 